$\log _{6}, 950 \mathrm{~g}$

UCRL-ID-120019

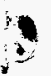

\title{
Research on Computed Tomography Reconstructions from One or Two Radiographs: A Report and the Application to FXR Radiography
}

\author{
Norman Back \\ Dan Schneberk \\ Charles McMillan \\ Steve Azevedo \\ Michael Gorvad
}

January 26, 1995

Work performed under the auspices of the U.S. Department of Energy by the Lawrence Livermore National Laboratory under Contract W-7405-Eng-48. 


\section{DISCLAIMER}

Tuls document was prepared as an acoound of work sponsored by an agency of the United States Goverament. Neither the United States Governmeat wor the University of Califorala nor ang of their emplogets, makes anj warrenty, express or implied, or assumes anj legal liability or responsiblity for the accuracy, completenex, or usefulaess of anj information, apparatus, product, or process disclosed, or represents that its use would not infringe privately ombed rights. Reference berein to anj specific conmercial products, process, or service by trade mame, trademark, manufacturer, or otherwise, does aot mecessarily constilute or imply its endorsement recommendation, or favoring by the Ualted States Goverament or the Uaiversity of Callforia. The views and oplnions of authors expressed herein do not necessirily state or rellect those of the United States Government or the Uaiverdity of Calffornia, and thall not be used for advertising or product endorsement purposes.

This report has been reproduced directif from the best available cops.

Available to DOE and DOE contractors from the Ofice of Scientific and Technical Information

P.O. Bax 62, Oak Ridge, TN 37831

Prices arailable from (615) 576-8401, FTS 626-8401

Available to the public from the National Technical Information Service

US. Department of Commerce 5285 Port Rojal Rd, Springfield, VA 22161 


\section{DISCLAIMER}

Portions of this document may be illegible in electronic image products. Images are produced from the best available original document. 
Research on Computed Tomography

Reconstructions from One or Two Radiographs:

A Report and the Application to FXR Radiography

\author{
Norman Back \\ Dan Schneberk \\ Charles McMillan \\ Steve Azevedo \\ Michael Gorvad
}

January 26, 1995

This paper is the final report for LDRD Project 93-SR-107

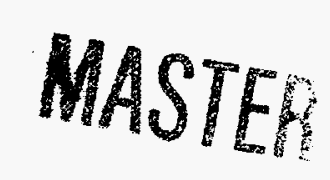




\section{Introduction}

II. Computed Tomography from 1-2 Views

a. CT reconstruction fundamentals

b. CT reconstruction alternatives for 1-2 view $\mathrm{CT}$

c. Measures of performance for 1-2 view CT

d. CT inspection performance against relaxed assumptions

e. The impact of additional views

III. Applying CT reconstruction methodology to FXR radiography

a. FXR radiography

b. Converting films to exposure and to attenuation

c. Using multiple films

d. CT reconstructions of FXR radiographs

IV. Summary \& Discussion 


\section{Introduction}

This report documents some cooperative research into volumetric image reconstruction from single radiographs. Imaging dynamic events is the most important application for this type of work, but the techniques have possible extensions. Two general objectives guide this work. The first objective is to gain an understanding of the assumptions and the limitations of single-view methods for representing internal features. Second, we endeavor to obtain and/or develop techniques for performing image reconstructions with FXR radiographs. If possible, we seek to obtain some quantitative measure of the accuracy of this class of image reconstructions in two respects: $i$ ) in terms of the dimensional accuracy of feature boundaries, and ii) as pertains to the accuracy of the voxel intensities. Dynamic events are not always self-calibrating, and it is important to establish the reconstruction accuracy of single-view methods for placing bounds on the kinds of conclusions which can be advanced from single-view reconstructed images.

Tomographic reconstructions from a single transmission radiograph will involve some assumptions concerning the object. The number of radiographic views recommended by the ASTM[ASTM] for performing high-fidelity CT is on the order of 1.5 times the number of detectors used to image the field of view in the horizontal direction for a rotate-only scan. The different views are acquired at different rotational angles of the object for 360 or 180 degrees. This recommendation usually leads to the acquisition of 750 to 1500 views, for detector arrays between 512 and 1024 in size (e.g., our Photometrics camera can acquire a 1024 by 1024 image, and CCD chips with dimensions of 2048x2048 are commonly available).

If the object has axial symmetry, the axis of symmetry can be identified, and we equate the axis of symmetry with the axis of rotation, all of the radiographic views from different view angles will be equivalent. Consequently, only one view is needed to perform the reconstruction. Axial symmetry is the key assumption for applying CT reconstruction algorithms to single radiographs, but other considerations are important. Even if axial symmetry is present in the object, and the axis of symmetry can be identified with minimum error, other information is important to the reconstruction process. It is important to know the beam shape: fan/cone, or parallel. The signal to noise ratio of the input data is crucial and will figure importantly in the overall image quality ( there will be no improvement derived from the additional number. of views). Also, it is important that the data be converted to attenuation units prior to reconstruction. Lastly, certain data analytic conditions are important: the presence/absence of errant pixels, and the amount of blur in the image. All of these factors will impact the accuracy of the image reconstruction.

Computed tomographic image reconstructions provide dimensional detail of internal structures of objects and provide a measure of the per-voxel attenuation of material in the object. When assumptions 
behind a reconstruction algorithm are not satisfied, or are satisfied in a limited way, the accuracy of the reconstructed image is compromised. Figure 1 illustrates different effects which arise when the algorithm and the data acquisition are not consistent. As seen in Figure 1, the errors in the reconstructed image can take on certain characteristic patterns referred to as artifacts. It is the goal of CT analysis to discern the 'real' features of the internals of an object in the midst of a certain level of artifactual content in the image. By understanding the ways in which CT reconstructions from a single radiograph can produce misleading results we hope to develop some measure of the benefits and limitations of single view techniques. 


\section{Computed Tomography from 1-2 Views}

Transmission based computed tomography scanners generate 3D measurements of attenuation for objects and assemblies. The quality of the tomographic image is a direct function of the properties of the input transmission data, and of the particulars of the scanning technique. In single view tomography, axial symmetry is important in two respects: i) if symmetry is present, all of the feature information for the object is contained in one radiograph, and ii) it is only necesary to reconstruct the center plane in the direction of the $\mathrm{x}$-ray beam (see figure 2 ) and use this image as representative of the entire 3D object. It is the goal of this section to examine the inspection performance of single-view reconstructions when the input data conforms to the assumptions, when the data includes small departures from required assumptions, and when the data contains large departures from the required conditions.

This evaluation unpacks into four tasks: i) present candidate CT reconstruction approaches for single view data sets, ii) sketch the fundamentals of the algorithms in enough detail to motivate our list of important assumptions, iii) describe the measures of reconstruction accuracy we will use throughout this section to evaluate the performance of algorithms and approaches, and iv) generate different input data sets with certain amounts of error and evaluate the impact on the reconstructed image. Simulated data will be used primarily in this section since the underlying assumptions for the input data can be perturbed in measured amounts, and different conditions can be inserted into the input data singly or in combination. From simulated data we can get a close look at the behavior of reconstruction algorithms for a particular condition, without the variety of effects usually present in acquired data. The simulation tools used here are documented in [AZEVEDO90].

\section{Ila CT reconstruction fundamentals}

The object of transmission-based computed tomography is to recover the object function from line-integral measurements through the object function. While there are many methodologies for performing this operation, the transmission data and the end result are the same. From the general statement of the reconstruction problem we will show the types of simplifications which arise for objects with axial symmetry and how this property relates to the methods of image reconstruction. It will also be important to present some description of computed tomography reconstruction artifacts, which arise from certain conditions in the input data, since single view reconstructions will in no wise be exempt from these kinds of problems.

The simplest description of CT reconstruction proceeds from the simplest input data, 'x-ray transforms', which are an idealized version of an X-ray attenuation projection of a 3D object function. The experimental analogue of this idealized construct is the transmission image of an object function by 
the 'primary beam' component which has been converted to 'attentuation units'[JOHNS82]. We assume a 2D flat detector which integrates the fluence over time and possibly energy. Given an $\mathrm{x}$-ray source at position, $s=(0,-D, 0)$, with some source distribution function $S(s, d, E)$ (which specifies an intensity for each source-detector pair location, at some or possibly a variety of energies $E$ ), some object function $f(v)$, where $v$ denotes a 3D vector, $v \rightarrow\left(v_{x}, v_{y}, v_{z}\right)$ (for this work the object function is in linear attenuation coefficients), a two-dimensional detector $P(d), d \rightarrow\left(d_{x}, d_{y}, d_{z}\right)$ oriented in a plane perpendicular to the emerging $x$-ray beam $\left(d_{y}=\right.$ object to detector distance), and with $\Omega$ representing the 3D support [GRANGEAT87] for the source-object-detector geometry, then the available intensity from the $\mathrm{x}$-ray transmission can be written:

$$
\text { (1) } P(d)=\int \Omega f(v) \delta(S(s, d, E)-v) d v
$$

The function $P(d)$ represents the image of the object attenuation as illuminated by the the source distribution function over the relevant 3D support of the object.

It is canonical to assume two conditions are satisfied by the ' $x$-ray transform'. First, that we are operating between the two limiting cases: i) perfect transmission through the object (none of the detected $x$-rays are attenuated by the object and there is no variation in $P(d)$ due to changes in object thickness), and ii) no transmission (infinite attenuation, all values in $P(d)$ are infinity). Second, we assume that $P(d)$ covers the object, or at least half the object, and includes a measure of the fluence outside the object (finite extent, i.e,some image of the boundary of the object ). If the source is an $x$-ray spot, a cone-beam source distribution can be assumed, and the $\mathrm{x}$-ray transform onto the detector is an integration along separate lines from a single source location to each detector element, as follows:

(2) $P(d)=\int \Omega f(v) \delta\left(\left(s+l_{d} u\right) \cdot v\right) d v$

where $\mathbf{u} \rightarrow\left(\mathrm{u}_{\mathrm{x}}, \mathrm{u}_{\mathrm{y}}, \mathrm{u}_{\mathrm{z}}\right)$ denotes the direction cosines of the line between source and detector (point $\mathbf{s}=$ $(0,-D, 0)$, and the points $\left.d=\left(d_{x}, d_{y}, d_{z}\right)\right)$, and $l_{d}$ is the path length between source and detector, for detector position $\mathbf{d}$.

If the source distribution is a set of parallel rays, the $\mathrm{X}$-ray transform is further simplified as follows,

(3) $P(d)=\int \Omega f\left(v_{x}, v_{y}, v_{z}\right) \delta\left(d_{x}-v_{x}\right) \delta\left(d_{z}-v_{z}\right) d v_{y}$ 
For parallel data, in these coordinates, all integrations take place along the y-axis, with the rows in the $P(d)$ image recording the transmission through the different $x-y$ planes in the object, indexed by $z$. Columns in the image record the transmission through the different $y-z$ planes indexed by $x$.

Both source distribution geometries, cone and parallel, are useful models of radiographic technique. There is certainly a problem with assuming the $\mathrm{x}$-ray source is a single point in space. $X$-ray sources possess some finite spot size. However, if the size of the spot is small relative to the detector element size, or the blur due to spot size can be reduced by demagnification, the cone-beam representation has proven to be a useful point of departure. Parallel beam data can be achieved in a variety of ways. First, if the source-to-object distance is large relative to the size of the object (cone angles less that 1.5 degrees), parallel assumptions have been shown to introduce very little error. These small cone angle techniques are a regular part of high-energy radiography for a variety of reasons. Second, parallel data can be obtained through some kind of coded-aperture arrangement. For the above reasons, and for sake of simplicity, we will restrict our discussion to parallel-beam and cone-beam data acquisition.

If the object has axial symmetry the last of the above equations can be further simplified. Without loss of generality, we can assume the axis of symmetry is the vertical z-axis in the coordinates described here (see figure 2). The symmetry of the object function is in the $x-y$ planes, and only the distance from the $z$-axis is required to describe the variation in the object. Now the object function is in two variables, $r$ and $z$, with $r=\left(x^{2}+y^{2}\right)^{1 / 2}$. If the data is parallel-beam the entire integral can be rewritten in one form of the Abel transform with the transmission through the $x-y$ planes, for each plane in $\mathrm{z}$, as follows:

$$
\text { (4) } P(x)=2 \int_{\mid x /}^{\infty}[f(r) r] /\left(\left(r^{2}-x^{2}\right)^{1 / 2}\right) d r
$$

The x-ray transform of an object, whether cone-beam or parallel beam, is considerably simpler if axial symmetry is assumed. If the beam is parallel the $\mathrm{x}$-ray transform is the Abel transform of the object function. It is important to note that cone-beams do not permit this convenient re-writing into the Abel Transform presented here. However, it may be the case that some reduction to a modified transform which includes the beam divergence described in equation (2) is possible.

Three different mathematical approaches to reconstructing the object function are in routine usage for computed tomography reconstructions in general and for reconstructing single-view input data in particular. The most widely used class of algorithms is based on the 'Fourier Slice Theorem' or 'Central Slice Theorem' which provides a means for relating the x-ray projections through an object to 
the Fourier transform of the object function[BARRETT81] [KAK87]. A less widely used and recently developed alternative based upon the first derivative of the 'Radon Transform' has shown some distinct performance benefits for radiographic techniques which include moderate to large cone angles [GRANGEAT87]. For axially symmetric objects the most common approach is to invert the Abel Transform described by equation (4) [HANSON82], thereby reclaiming the object function from its projections. Each mathematical approach has given rise to whole classes of reconstruction algorithms, which vary in the number of computations (single-pass or iterative), the space in which the object function is composed (Direct Fourier Inversion techniques or Backprojection), and in the way each handles different source geometries and object movement regimes.

For this report we will restrict our attention to the first and third approaches. This expresses no opinion on the merits of the Radon transform approach, which has been shown to provide decided performance advantages in many circumstances[GRANGEAT88]. Rather, this simply reflects the state of the research performed up to this point. Also, many in-depth comparisons have been performed with the 'Central Slice Theorem' approach, and the differences are quite well known [AZEVEDO91b]. Many good texts exist on the details of the different algorithms, and there is no attempt here to be redundant. Our approach will be to sketch the basic ideas of the algorithms in sufficient detail to motivate the measures of inspection performance we will use, and to highlight possible weaknesses in the implementations for acquired data.

\section{II.b CT reconstruction alternatives for $1-2$ view $C T$}

We will sketch the two different algorithms in reverse order of the list presented above for reasons that will be made clear. In each case, we present the central idea of this reconstruction algorithm, give one formula, or set of formulae, for a discrete implementation of the algorithm, and discuss some the the issues involved in performing reconstructions in this manner.

Each class of algorithm includes different specific implementations according to the type of acquisition geometry, and according to the particular representation of the x-ray transform chosen. While a number of different regimes exist for obtaining the required data, it is the 'third-generation' (rotate-only) scanning technique that is particularly interesting for objects with axial symmetry. For this modality, the axis of symmetry can be regarded as the rotational axis, which is normal to the x-ray beam, and then every view is the same. One consequence of identifying the axis of symmetry with the rotational axis is the problem of a possible 'tilt', if the axis of symmetry is not normal to the $\mathrm{x}$-ray beam. The radiograph acquired with a 'tilt' will not lead to symmetric radiographs from all rotational angles. Consequently, the assumptions of the reconstruction routine will be violated, and the reconstructed image will be misleading. We will revisit this issue in the next section. 
The Abel transform representation and its inverse can be used to reconstruct the center slice of the object function. This method was first discussed and implemented by Ken Hanson at Los Alamos National Laboratory. The discrete implementation is computationally fast, and performs correct reconstructions for parallel data. An LLNL implementation of this technique has also shown marked speed advantages over other algorithms.

The key idea of the Abel Transform algorithm is the implementation of the inverse transform. Given the representation of $x$-ray transforms of axially symmetric objects as Abel Transforms in (4), the object function can be reclaimed by computing the inverse transform[BARRETT81]:

(5) $f(r)=-(1 / \pi) \int_{r}^{\infty}[d P(x) / d x] /\left(\left(x^{2}-r^{2}\right)^{1 / 2}\right) d x$

The discrete implementation of this method can take two forms:either the inverse transform is calculated according to equation (5), or a discrete derivative is approximated by using the geometry of the strip integrals of a sphere. In the first algorithm, a derivative is calculated over the horizontal axis of the projection data, with an appropriately designed filter, and the data is then weighted by position. In the second implementation, a voxel at a particular radius is reconstructed by subtracting the attenuation of the outer rings, and taking into account the ratio between the area of a tangent strip on a circle and the voxel in the reconstructed cube. For the second approach the reconstruction takes place by calculating from the outside shell to the center, subtracting the cumulative value of the reconstruction performed up to that radius While the first implementation is strictly limited to parallel data, the second procedure could possibly be extended to a cone, since strip integrals of a sphere have a direct computation for any geometry. For a variety of data analytic reasons, the second approach is more widely used. One discrete representation of the form of the algorithm is as follows:

(6) $f\left(r_{i}\right)=\left[P\left(r_{i}\right) * w\left(r_{i}\right)\right]-\Sigma_{j=1}\left[P\left(r_{i+j}\right) * w\left(r_{i+j}\right)\right]$

$$
\text { and } f\left(r_{\max }\right)=P\left(r_{\max }\right) * w\left(r_{\max }\right)
$$

where $w\left(r_{i}\right)$ is the radius specific weighting of the voxel in that chord to the total chord volume in that spherical shell.

The advantage of speed mentioned above could be significant. Iterative methods for obtaining better reconstructions are now an interesting possibility. This has been pursued by Hanson [HANSON82], and elsewhere by Dinton[DINTON90], with interesting results. Also, smaller lessexpensive computers can handle large radiographs, facilitating the widespread use of this algorithm wherever appropriate. 
The second reconstruction approach utilizes the class of reconstruction algorithms based on the 'Central Slice Theorem' or 'Fourier Slice Theorem'. There are no assumptions on object shape intrinsic to the reconstruction algorithm. Axial symmetry is not a part of the reconstruction algorithm; rather, this assumption is used to generate the other rotational views. By separating the characteristics of the object function from the reconstruction algorithm this approach can make use of additional information known about the object or the data for generating all of the other rotational views.

This class of algorithms is based on a mathematical relationship between the projections through an object function and specific lines in the 2D Fourier transform of the object function. Stated in many different forms, the central slice theorem establishes an equivalence between the Fourier transform of a projection through the object function at a particular rotational angle, and a line intersecting the origin of the 2D Fourier transform of the object, represented in polar coordinates. The rotational angle of the projection is the radial angle of the line in 2D Fourier space[BARRETT81] [KAK87]. This equivalence gives rise to two different non-iterative reconstruction algorithms, i) the direct Fourier inversion algorithms (DFI), where the projections are used to fill up the 2D Fourier transform, the object function being reclaimed by an inverse transform, and ii) the convolution back-projection algorithms (CBP), where each projection is filtered by a filter function which emerges from the central slice theorem, and the filtered projections are summed (back-projected), as that pixel intersects with that voxel in the source-detector geometry.

For parallel data we employ filtered back-projection, the convolution back-projection algorithm for fan-beam geometry, and for small cone-angle data sets we use the Feldkamp algorithm, which is the 3D generalization of convolution backprojection for a cone-beam geometry. In this approach each radiographic view is convolved with an apodizing filter, and the filtered data is back-projected onto the reconstructed image. Source-to-object distances (sod) and source-to-detector distances (sdd) are used explicitly to calculate the intersections between the transmission line integrals and the reconstructed voxel. This is the most common cone-beam reconstruction methodology found in industrial scanners and has been the subject of numerous investigations[KAK87].

There are three steps to the Feldkamp algorithm which are important, filtering the projection data, performing the back-projection along tilted fans, and applying weights to the reconstructed results. One formula for implementing the Feldkamp algorithm is the following:

$$
\text { (7) } f(v)=\int 2 \pi \operatorname{sod}^{2} /\left(\operatorname{sod}-d_{x}\right)^{2} \int{ }^{P_{\theta}}(d)\left(\operatorname{sod} /\left(\operatorname{sod}^{2}+d^{2} x+d^{2} z\right)^{0.5}\right) h\left(s^{\prime}-d_{x}\right) d x d \theta
$$

where: sod - is the source to object distance $P_{\theta}(d)$ - denotes the projection data ( scaled to the center of the object) $\mathrm{h}()$ - denotes the filter function 
$\mathbf{v}$ - the 3D position in object space, and $v_{x}$ the component in the $x$ direction d - the 3D position of the detector with $d_{x}$ and $d_{y}$ the $x \& y$ components

$\mathbf{s}^{\prime}$ - denotes the intersection of the ray passing through voxel $\mathrm{v}$ with the detector plane translated to the center axis, $s^{\prime}=\left(r \cos (\theta-\phi) /\left(1+r \sin (\theta-\phi) / s^{\prime} d\right)\right.$,

where

we have represented the $x, y$ coordinates in polar form $(r, \phi)$ for convenience.

The three steps are nested in the above formula, the backprojection inside the filter, the filtering applied to the weighted pixel values, and the entire projection weighted by the distances in the source-object geometry. The implemented algorithm will exchange discrete summation signs for continuous integrals, but, since this is a coverage of key concepts, they were left in for similarity with the earlier equations.

It is important to notice the character of the reconstructed voxel. First, each voxel is a weighted sum across the projection data. Consequently, additional fidelity is gained from the additional number of views, with a noise reduction on the order of $1 / \mathrm{sqrt}$ (\# of views). The elements of the sum are calculated according to the intersection of the ray-path through the voxel and the detector plane (translated to the origin or left in the original distance), which follows the lines of the source distribution. Weights are calculated from the solid angle relationships in the cone beam distribution. The exact form of the filter applied to the projections and its row-wise application follow from the Fourier Slice Theorem covered in detail in [KAK87]. For a particular voxel, indexed by $(r, \phi, z)$, reconstructing fan/cone beam data with a parallel algorithm will incur two types of errors: $i$ ) incorrect weighting of the projection data, since in parallel data the solid angle apertures are all the same, and ii) the intersections with the detector plane, $r \cos (\theta-\phi) /(1+[r \sin (\theta-\phi) /$ sod $]$, will not be precisely true, although this will involve less error for asymmetries not on the center plane. Notice that as sod becomes large the data becomes more and more parallel, so the size of both of these errors is a direct function of cone angle.

Using an in-house version of the Feldkamp code, 'fkrecon', as the basic reconstruction methodology, three different techniques for generating the other rotational views were developed and implemented: 'fksymm', 'fksingle', and 'symmod'. Each code differs in the way in which the missing views are generated, and in the means by which other information concerning the object can be utilized. The 'fksymm' code is a direct modification of 'fkrecon' where the backprojection operation is forced to use a single radiographic projection. No interpolation is applied to the rotational views not acquired. This code strictly enforces the assumption of axial symmetry. This code reconstructs fan/cone data for small cone angles, but will not perform well on asymmetries. As such, it can be used to isolate the effects of asymmetries on the CT reconstructed image.

The second code, 'fksingle', is our first means for including some information on asymmetries in the single view reconstruction process. 'Fksingle' makes explicit use of the knowlege of the position of 
the axis of symmetry obtained by a priori means. If the axis of symmetry is known, the view from 180 degrees can be calculated by flipping the image about this axis. Using the single view as the 360 degree view, all of the other rotational views are calculated by interpolating from this two image set. At this point in time our research has shown a slight preference for squared cosine and squared sine weighting, a practice first employed elsewhere[LANSFORD], over a bi-linear interpolation between the views. However, the advantages of this interpolation scheme compared to bi-linear techniques is not large. Once all of the views are calculated the reconstruction is performed with 'fkrecon'.

In detail, the reconstruction process for this approach is as follows. From a knowlege of the center axis of symmetry, interpolate a set of angular views which matches a third generation scan as follows:

$$
P_{180}\left(d, c_{r}\right)=f l i p\left(P_{0}\left(d, c_{r}\right)\right): P_{0}\left(d, c_{r}\right)
$$

and for any $\theta$, calculate $P_{\theta}\left(d, c_{r}\right)$ as

$$
P_{\theta}\left(d, c_{r}\right)=\cos ^{2}(\theta / 2) P_{0}\left(d, c_{r}\right)+\sin ^{2}(\theta / 2) P_{180}\left(d, c_{r}\right)
$$

where $c_{r}$ has been identified to emphasize the importance of the knowlege of the axis of symmetry for this method of image reconstruction.

The 'fksingle' approach to generating the other rotational views is not an exact technique for handling the asymmetries. First, for cone-beam data, the calculated 180 degree view will be precisely true only if the asymmetries are positioned at the source to object distance. In this case the effect of the superposition of the different structures in front of, and behind, the asymmetries, is exactly the same regardless of direction. If the asymmetries are closer to the x-ray source, or closer to the detector, a horizontal flipping of the radiograph will not precisely reflect the different magnifications. For parallelbeam data (no magnification), the flip operation is exactly the 180 degree view. Secondly, for any type of data, the trigonometric weighting is not entirely accurate, and the interpolated views do not trace out the correct path for the rotation of the asymmetry in the object matrix. It needs to be re-iterated that behind all of these operations is the choice of the axis of symmetry, which, if incorrect, biases every single pixel in the reconstructed image.

The 'symmod' code can make explicit use of model-based information concerning the symmetric and asymmetric information in the radiograph to compose the other rotational views in a more exact fashion. Different means are used to calculate two images: i) an image of the symmetric part of the radiograph, and ii) an image of the asymmetries. The non-zero features in the asymmetries image are internally rotated (each feature is shifted in the projection), according to the selected angular increment. The amount of shift is dependent on the assumed position of the asymmetry and the feature shape. 
Without any information on the asymmetries the 'symmod' code assumes all features are at the maximum radius from the center of the object, and are in the shape of spheres. However, a number of different assumptions concerning feature shape could be considered. This approach is capable of making use of more ancilliary information about the object than the other two approaches.

The equations for the 'symmod' approach are as follows:

$$
\begin{aligned}
& P_{\theta}\left(d, c_{r}\right)=P_{\text {sym }}\left(d, c_{r}\right)+P_{\text {asym }, \theta}\left(d, c_{r}\right) \\
& \text { where } P_{\text {asym }, \theta}\left(d, c_{r}\right)=\operatorname{shift}\left(P_{\text {asym }, 0}\left(d, c_{r}\right)\right) \text { and } \\
& \text { shift }=(r \cos (\theta-\phi) /(1+[r \sin (\theta-\phi) / \text { sod }]) \text { is applied to the non-zero elements of } \\
& \quad P_{\text {asym }, 0}\left(d, c_{r}\right) \text { and } \theta, \phi \text { defined as before }
\end{aligned}
$$

We will make use of these three codes in the simulation work which follows. The 'fksymm' code will be used to examine the effects of asymmetry by strictly enforcing symmetry in the reconstruction process. Also, we will use 'fksymm' to evaluate the effects of reconstructing fan/cone data with parallel assumptions, independent of problems with asymmetries. We will examine the performance of the 'fksingle' code for general purpose single-view reconstructions in the presence of some fraction of asymmetry. This approach includes some limited means for handling asymmetries, and requires very few assumptions concerning the asymmetric features (unlike 'symmod'). It will be important to determine the performance of this code against different types of asymmetries in preparation for possible use on FXR radiographs.

Evaluating the performance of these algorithms in a quantitative way requires data which includes measured amounts of departures from underlying assumptions. As mentioned above we will use simulated data since the different effects we are interested in can be inserted in particular locations in measured amounts. While this is arguably the correct methodology for evaluating algorithms against required assumptions, it strays from the more standard measures of the CT reconstruction performance advanced by the ASTM[ASTM]. There are good reasons for this; the 'standard' measures of performance assume an accurate algorithm, but this is exactly what is at issue in single-view reconstruction work. We need to specify measures of reconstruction accuracy which identify the presence of errors due to the violations of assumptions, and we will discuss these choices in the next section.

Before proceeding further we need to address a general question posed to the activity of performing single view CT reconstructions. What are we really doing - some fancy re-arrangement of the same data? Strictly considered, only perfect axial symmetry will result in exactly correct CT reconstructions. Consequently, what can be learned from a CT reconstruction that is not observable in the radiograph? If there are asymmetries in the radiograph, then the assumptions for CT are violated and it will give you incorrect results. If symmetry is true, then maybe there is no point. However, CT 
reconstructions present the structure of the center of the object, given the projection. It is not clear if human viewers can perform this operation accurately. Certainly there is no 'new' information in the CT reconstruction that is not in the radiograph. But single-view CT reconstructions do make serious and decided use of the symmetry in the image, and generate an internal view of the object which assumes that symmetry is indeed true. Maybe the better questions are: do CT reconstructions present a view which is useful in your analysis? and could you spot an artifact due to a problematic assumption if you saw one? We will attempt to return to these fundamental concerns in the course of analyzing the CT reconstructed data.

\section{II.c Measures of performance for $1-2$ view $C T$}

The goal of single view CT inspections is to enable an examination of internal features of an object or assembly. Measures of performance should help in establishing some confidence (or lack of) for the conclusions which emerge from the analysis of the object. Single-view CT requires more assumptions than CT inspections with the full complement of views, and we need to develop metrics for measuring the degree to which those assumptions are in effect, as well as for measuring the accuracy of the CT reconstructed image. In this section we are assessing algorithm performance with known errors in simulated data. We seek to identify the characteristic patterns in the reconstructed images which stem from the violation of some assumption, and plot the prominence of those artifacts as the departure from the assumption grows. Our measures of performance need to measure different aspects of reconstruction accuracy, not the crispness of edges. Crisply defined edges in a reconstructed image in single view tomography may be crisply wrong.

In order to assess reconstruction performance for basic questions of accuracy our simulation tools do not need to model all the physical processes which occur in the interaction between penetrating radiation and an object. We need two capabilities, i) the ability to generate a pristine radiograph which includes some quantitative level of a particular condition, and ii) the ability to generate the perfect simulated image which does not include the malady. The CT project has developed a number of simulation tools for detecting misalignments in scanners, of which two codes will be useful here: SIM and MKIMAGE. The SIM code generates projections through volumes which are discrete implementations of equation (2), perfect cone beam projections. The source, detector elements, and object(s), can be placed anywhere in space, at any orientation, so the full scope of misalignments and source-detector geometries can be implemented. The second code, MKIMAGE, makes the perfect volumes which should be the result of reconstructing the data in SIM. The same input file drives both codes ensuring the same object space for both images. Combined with a reconstruction code, SIM and MKIMAGE form a complete set for performing reconstruction algorithm development work.

Three measures of performance are usually regarded as important for evaluating CT reconstructed images: i) the FWHM of the estimated point spread function, which is a measure of the 
spatial resolution in the image, ii) contrast-dose-detail calculations, which are another measure of contrast sensitivity, and iii) the voxelwise variance, which includes a number of different components. The voxelwise variance includes three components, i) noise due to photon statistics, ii) systematic errors stemming from detector biases, or misalignments, and iii) the intrinsic variation of the object. One goal of CT analysis is to isolate the third component in the voxelwise variance independent of the first two. The first two CT performance measures are not oriented towards the characterization of errors in the reconstructed image, but are more concerned with the fidelity of the image. We are concerned with reconstruction accuracy, and variations on the voxelwise variance are more suited to this task.

Knowing that the simulated data has been perturbed in some precise way, we are interested in the accuracy of the algorithm in three respects: i) overall accuracy, i.e., what is the level of error which is in the entire image given this level of perturbation of the basic assumptions; ii) local accuracy, i.e., is there a particular area in the reconstructed image which is particularly problematic for this type of problem with this input data; and iii) what is the maximum error in this reconstructed image. This last measure places a bound on just how inaccurate the image can get, and by implication, how wrong the interpretations can be. Following the standard practice in single-view reconstructions, only the center slice will be used in the comparisons. All measures of performance will derive from the difference between the 'correct' image and the image calculated from the perturbed assumptions. Either the 'MKIMAGE' code will supply the 'correct' image, or we will obtain a reconstructed image from a data set where all the assumptions are satisfied.

The overall accuracy will be measured by the root-mean square ( $\mathrm{ms}$ ) difference between the 'correct' image and the reconstructed image which includes a pertubation of some assumption. Local accuracy will be measured in two ways: i) the rms error calculated on a rectangular portion of the image; ii) the rms error at some circular extracted region to measure error as a function of radius; and iii) the maximum absolute value of the difference between the 'correct' and perturbed images. The circular extraction statistics will be important when it is expected that the error is correlated with the cone angle in the plane of reconstruction.

The measures of performance are important in themselves but the real target in this investigation is to consider the impact on the interpretations of the data. Computed tomography images are used to identify different materials (as they differ in attenuation) and provide dimensional measurements of internal features. The accuracy of the reconstructed image directly impacts the ability to perform this task. Consequently, we will attempt to trace the impact of the values of the different levels of inaccuracy on the joint task of making dimensional measurements and identifying different materials in the CT reconstructed images. We will try to be sensitive to the variation of this error with position in the image if that turns out to be important.

IL.dCT inspection performance against relaxed assumptions 
Our full list of assumptions for single view reconstructions identifies four different aspects of object and technique requirements. First, and foremost, the object has axial symmetry, in particular, symmetry with respect to the vertical axis (the z-axis), as pictured in figure 2. Second, and related to the axial symmetry in the object, is the measured position of the axis of symmetry in the radiograph. Two characteristics of the technique are involved here: $i$ ) the axis of symmetry in the object is perpendicular to the X-ray beam; and ii) the position of the axis of symmetry is measured with minimum error. Third, as in the multiple-view case, accurate tomographic imaging requires that the source-detector geometry be correctly reflected in the reconstruction algorithm. This includes the requirement that the center axis of symmetry and the center of the $x$-ray beam be in the same location. The fourth consideration, noise, or noise level, is a problem for single-view work since there will be no noise reduction from the acquisition of the different rotational views, and it could overwhelm whatever detail is present in the reconstructed image.

The first two assumptions span a number of different mechanisms for obtaining asymmetries in the acquired radiographs. The object or event might have a small asymmetry at some location, or it might possess a tilt in the axis of symmetry relative to the $\mathrm{x}$-ray beam, which can result in a preponderance of asymmetry in the entire radiograph. In a similar fashion, if there is an error in the identification of the position of the axis of symmetry in the radiograph, the reconstruction will not reflect the symmetry in the object. This last source of error is particularly pernicious, since small errors in the identification of the center of rotation, or lack of concurrence between the center axis of symmetry and the center of the $\mathrm{x}$-ray beam (mid-line offsets for the cone-beam case), can result in decided inaccuracies for even small errors [SCHNE91].

Regardless of the means by which it occurs, a violation of the axial symmetry assumption results in a radiograph with a certain amount of asymmetry. The problem may be in the object, or it may be in the technique, or in the measurement of the axis of symmetry. From a single radiograph it is difficult to discern the different origins of the observed asymmetry. However, the amount of asymmetry can be measured directly from the radiograph - divide the image in two at the axis of symmetry, flip one of the half-images horizontally, and calculate the sum of squares of the difference (or the sum of the absolute values of the differences, if those statistics are found to be more useful). Care must be taken in flipping the image, and a consistent policy adopted for dividing the image on pixel boundaries or interpolating in some fashion. However, provided the method is applied consistently, relative amounts of asymmetry between radiographs will be correctly identified.

The amount of asymmetry in any acquired radiograph will vary with the nature of the features in the radiograph, but we can reduce these to a few cases for simulating specific effects. The asymmetries will be sharp high-contrast features or low-contrast features. The asymmetries will vary in their position, and will be of different sizes. We have determined two simulation phantoms which include a number of 
different asymmetries which will be used to cover the gamut of different cases: i) a high-contrast phantom, and ii) a low-contrast large-area phantom. Figure 3 includes the 'MKIMAGE' center slices of these two synthetic assemblies.

Both phantoms include two different types of objects, those which are symmetric about the zaxis and those which are not. In both cases the symmetric component constitutes the greater proportion of nearly every chord through the object. In the high-contrast phantom, all the asymmetries are spheres at different radii from the center axis of rotation, and they have no attenuation. Spheres were selected to make the computation of total asymmetry in the volume more convenient. The sizes and radii of the spheres were chosen to evaluate the impact of the position of an asymmetry on the error fraction in the reconstruction, as well as the overall level of asymmetry. Figure 4 contains some of the examples of the different patterns of asymmetry we will consider for the high-contrast phantom.

The asymmetries in the low-contrast phantom are all low-attenuating large ellipses at different heights, which are shifted at increasing radii from the center. The ellipses are long in the horizontal dimension, and the different levels of asymmetry are achieved by moving the centers of the ellipses in alternating distances from the center axis of rotation. Figure 5 contains some of the asymmetric patterns and is annotated with the corresponding level of asymmetry in each simulation. In this case the level of asymmetry can get quite large before the object fails to intersect the axis of symmetry. It will be important to compare the results of the low-contrast case to the high-contrast results with respect to total error vs. total level of asymmetry. For all of the asymmetry work we will reconstruct with 'fksymm', in order to measure the amount of error propagation due to asymmetries in the reconstruction process. We will also reconstruct with the 'fksingle' code to evaluate the improved accuracy which may result from a simple approach to asymmetries which makes the maximum usage of the knowlege of the axis of symmetry.

Error from reconstructing with the wrong source-detector geometry will be evaluated with a symmetric phantom which includes a moderate amount of internal detail. Figure 6 includes an image of the center slice, and different images obtained at different cone angles. We will employ the 'fksymm' code in this work, exclusively, since this code does accomodate cone beam geometries to some extent. Parallel reconstructions will be performed with 'fksymm' as well, where we will make the source to object distance very large, reducing the total cone angle to less than 0.1 degree. The motivation for using ' $f k s y m m$ ' in this dual role is that we can better examine errors due to source-detector geometry, independent of possible differences between interpolation schemes in different codes.

The impact of noise can be addressed with any of the above images; we will add different amounts of noise to some of the simulated transmission images in the previous work, and assess the impact. It will be important to examine the places in the image where noise accumulates. In this work we will use 'fksymm' and our in-house version of the discrete Abel transform approach. We will present these results with only a few comments. 


\section{The impact of asymmetries on single-view reconstructions}

We analyze the impact of high contrast localized asymmetries first. Fourteen different simulations were performed. The total amount of asymmetry was varied by including a larger or smaller number of the spheres in the simulation. Also, the spheres were configured to obtain the same level of total asymmetry, so that we could evaluate the error propagation due to the position of the asymmetry. Figure 7 is a plot of the total reconstruction error from the different simulations with the 'fksymm' code, and with the 'fksingle code' (we have used the center slice of the 'mkimage' volume for the 'correct' image). First, notice the variation in the reconstruction accuracy at the different total levels of asymmetry for the 'fksymm' results. Asymmetries that are at larger radii from the axis of symmetry will generate more error in the single-view reconstruction. Second, except for the position of the asymmetry, the total level of error in the reconstruction grows in near linear fashion with the total level of asymmetry; there do not appear to be multiplicative or special effects accruing as asymmetries get more prominent (indicated by the ratio of standard error to total level of asymmetry). Figure 8 includes images of the difference between correct and reconstructed images for the 'fksymm' work showing the localized character of the error, which is consistently a streak from the position of the asymmetry in towards the center of the reconstructed image.

The 'fksingle' approach results in a reduction of the total reconstruction error. A reduction of at least a factor of 2 is expected, since the operation of flipping the image to obtain the 180 degree view will eliminate the 'shadowing' of asymmetries produced by the 'fksymm' code. On average the total reconstruction error is reduced by 2.3 , which indicates that the trigonometric weighting is of some benefit in the interpolation of the missing views. Figure 9 contains difference images for the 'fksingle' reconstructions, showing the same position of errors and a streaking towards the center axis of symmetry, but at a lower overall level.

For both approaches the maximum error is substantial. Figure 10 contains plots of the maximum absolute difference for the 'fksymm' and 'fksingle' images. The values are only slightly less for the 'fksingle' code, which indicates that asymmetries are problematic for single view reconstructions under any circumstances.

For the low-contrast large asymmetries the reconstruction error is markedly less. For either the 'fksymm' or the 'fksingle' approach the reconstruction error is smaller for the low-contrast data than the error arising from same amount of total asymmetry in the high contrast case. Figure 11 contains radiographic and reconstructed images using the fksingle code. It is important to notice the lower absolute level of error for the 'fksingle' results.

Maximum absolute error follows the total error results for the low-contrast simulations. The errors are less for the low-contrast asymmetries. When the ellipses are shifted outwards in the horizontal 
plane to the point of no intersection with the center axis of rotation, the errors at the edges of the asymmetries increase markedly. Compared to the maximum absolute error reported in the high contrast simulations, the low contrast results involve less error.

Summarizing, high contrast asymmetries are the most problematic, and the errors get worse with increasing distance from the axis of symmetry. Low contrast asymmetries produce less error, as expected. Indeed, those asymmetries with a shape aspect in the direction of the plane of rotation about the axis of symmetry constitute the smallest problem for symmetric single-view methods. The 'fksingle' approach always produces less error in the reconstructions, when there are asymmetries, and converges to the symmetric case as the object possesses more axial symmetry. The 'fksingle' approach performs especially well for the low contrast asymmetries with shapes similar to the symmetric structure.

All asymmetries were placed at the position of maximum radius in the simulations (on the center plane). This is the optimal case for single view methods. When single view methods reconstruct asymmetric data, any asymmetry is always reconstructed into the center plane, regardless of its real 3D position with respect to the center plane. This is simply a result of the fact that transmission radiographs overlay complicated structures which are at different path lengths in the beam. If there is a large degree of asymmetry in a radiograph, and it is suspected that the position of the asymmetry has a vector component in the direction of the $\mathrm{x}$-ray beam, then single view reconstructions will provide results which are more misleading than the results presented here. This will get more or less problematic depending upon the contrastive properties of the asymmetry - high or low contrast. The presence of uncontrolled asymmetry should place severe bounds on the kinds of claims which can be made concerning any conclusions about dimensional measurements or feature identification in radiographic data.

\section{Error from reconstructing with the wrong geometry}

There are only two relevant geometries in this section, parallel-beam and cone-beam geometries. The different simulated radiographs were generated with different amounts of cone-angle divergence ranging from cone angles of 0 to 12 degrees in half-degree steps, some 26 different simulated symmetric radiographs. All of the reconstructions were performed with 'fksymm', once with the correct cone angle, and once with the 0 degree (less than 0.1 degree) angle, so as to provide a parallel reconstruction using fan beam data. Figure 12 contains plots of the total error in the reconstruction, and the maximum error, as a function of cone angle. Figure 13 contains images of parallel reconstructions, a cone beam reconstructions, and the difference images from the reconstructions for 3,6, and 9 degree cone angle data..

A number of conclusions can be drawn from the results in the figures. The total error in the reconstruction is not large when the small cone angle data (angle $<5$ degrees) is reconstructed with 
parallel assumptions and only the center plane is reconstructed. The maximum difference plots show that reconstruction with the incorrect geometry can result in large absolute error in selected parts of the image. The difference image explains the nature of the data in these two plots; as expected, the errors in the image grow with cone angle, and the level of this error is correlated with increased distance from the center of rotation.

\section{The impact of noise}

Noise in radiographic images inhibits clear imaging of details and confounds diagnoses and interpretations based on the images. In CT, with convolution backprojection or direct Fourier reconstructions, each reconstructed voxel makes use of some of the data from each rotational view. The resultant reduction in noise follows for the full-view CT reconstruction. In single-view CT, there are no additional views. This is particularly problematic due to the inherently noise-amplifying nature of CT reconstruction algorithms. The operation of CT reconstruction calculates the small amount of attenuation in a voxel from the comparatively large amount of attenuation in an entire line-integral. Noise enters the problem as a proportion of the line-integral, and it can be crucial just how the noise figures into the reconstructed voxel. For instance, the center voxel may be one part in 512, or 1024, of the entire line integral, but the noise can be one or two parts in 100. With high attenuating assemblies and large arrays the potential for overwhelming the attenuation in the small voxel is very great. Unfortunately, the unfolding of line integrals into small voxels is exactly why $\mathrm{CT}$ reconstructions are performed.

To assess the impact of noise in the presence of other problems, different levels of noise were added to the one phantom with a set of high contrast asymmetries inserted. The 7.2-degree cone sourcedetector geometry was employed and 'fksingle' used to illustrate the impact of noise. Levels of noise from 0.5 to $5 \%$ of the ray sum value were generated in 0.5 percent increments. Accuracy is the most important critierion here, so the standard error statistic, divided by the mean reconstructed value, was considered the most important to assessing reconstruction performance. The standard error was calculated using a 128-view no-noise reconstruction as the 'mean' value in the sum of squares computation.

Figure 14 contains a few radiographs and reconstructions from this series of simulations. Notice how the noise accumulates on the center voxel in the reconstructed image. In convolution backprojection each reconstructed voxel is a sinusoidal sum through the filtered radiographic data. For single view reconstructions, this means that the centermost voxels receive the impact of the filter function, but get very little noise reduction from other pixels. The formula for the variance of the noise as propagated through the CBP approach is presented below. 
(11) $\sigma_{\mathrm{v}}{ }^{2}(v)=\int 2 \pi\left[\operatorname{sod}^{2} /\left(\operatorname{sod}-d_{x}\right)^{2}\right]^{2} \int \Omega \sigma_{T}{ }^{2}(d)\left[\left(\operatorname{sod} /\left(\operatorname{sod}^{2}+d^{2}{ }_{x}+d^{2}{ }_{z}\right){ }^{0.5}\right)\right]^{2} h^{2}\left(s^{\prime}-d_{x}\right) d x d \theta$

where $\sigma_{\mathrm{r}}^{2}$ is the variance of the line integral, and $\sigma_{\mathrm{v}}^{2}$ is the variance of the reconstructed voxel Practically, this says to reconstruct the variance of each line integral with the square of the filter function.

For Abel-transform codes, noise amplification occurs through the process of subtracting an ever increasing sum from a smaller and smaller residual. The noise is accumulating in the sum as each shell is subtracted from the ray-sum at that decreasing radii. At the same time the reconstructed voxel contains proportionally more and more error. Figure 15 contains images of reconstructions with different approaches for a radiography with $5 \%$ noise. The variance of the noise as propagated through the Abel transform approach is presented in the next equation. Both of these results have assumed each pixel is an identical and independently distributed random variable (iid), which may not be strictly true given the wide scatter distributions present at high energies.

$$
\text { (12) } \sigma_{v}^{2}\left(r_{i}\right)=\sum_{j=0}^{\max } w^{2}\left(r_{i+j}\right) * \sigma_{r}^{2}\left(r_{i+j}\right)
$$

where $\sigma_{\mathrm{r}}^{2}$ is the variance of the line integral, and $\sigma_{\mathrm{v}}^{2}$ is the variance of the reconstructed voxel.

Figure 16 contains a plot of the standard error (divided by the mean) results for the reconstructed image vs. the level of noise input to the radiograph. It is important to note the non-linear aspect of the curve. From the images we can see that there are definitely areas where some detail can be deciphered in the image. However, the overall level of accuracy in the reconstructed image is seriously degraded. As expected, the effects of the noise are worse in the center of the reconstructed image. Unfortunately, many times this is an area of keen interest, since this is where the difference between the attenuation radiograph and the CT reconstruction is the largest.

A number of means exist for remediating the noise, such as smoothing beforehand, or possibly tapering the data before the reconstruction. Recent results have shown a simple smoothing kernel of 5 pixels can significantly reduce the noise buildup in the middle of the image for typical levels of noise to signal, 1-2\% [WINER]. For convolution backprojection, several different filters have been developed which handle noise less explosively than the canonical RAM-LAK filter which is implicit in the Fourier Slice Theorem. Both procedures will remediate the noise, but both sacrifice spatial resolution. This situation is not uncommon, since spatial resolution and contrast are always tightly related in any radiographic or CT image, and processing procedures which alter the spatial properties impact the contrast. The difficulty here is the amount of increase in the noise to signal ratio, which can be as high as a factor of $\mathbf{2 0}$ to $\mathbf{3 0}$ for typical noise levels, if no smoothing is applied. Of course the situation gets worse if there are other problems, such as small asymmetries, incorrect source-detector geometry, etc. 


\section{Il.e The impact of additional views}

There are two important reasons for acquiring additional views: $i)$ obtaining equal spatial fidelity in all directions in the reconstructed image, and $\mathrm{ii}$ ) reducing the noise in the reconstructed image by sqrt(number of views). The first issue addresses the question, how many views do I need to get all the spatial information in the detector array. As shown in [BARRETT81], if spatial blur is such that each detector of the $\mathrm{N}$ measurements has full spatial modulation, then $\pi / 2 * \mathrm{~N}$ views will carry that fidelity into the reconstructed image. There is a relationship here with distance from the center axis of rotation. For the same spatial fidelity, fewer detectors can be equated to less area to be reconstructed. In a different way, if the acquired image has less spatial fidelity than the sample spacing, the array can be resampled to the effective spatial resolution (i.e., fewer detectors), and fewer views are required. Consequently, the correct number of views is always for a certain radius and a certain spatial fidelity in the object space.

As seen in the last section, noise can be a problem in single view reconstructions. Additional views always remediate the noise in the reconstructed image. Indeed, there is no limit to the benefit of the number of views, which reduces the size of the noise variance by the inverse of the number of views [KAK87]. However, the benefit of the next view is proportionately less than the view before it. In this case the question is not the optimum number of views, but how many need to be acquired to obtain the fidelity necessary for a certain confidence level for a diagnosis.

To illustrate these relationships a high contrast asymmetric phantom was reconstructed with $1,3,6,9$, $12,15,30,60$, and 128 views. Figure 17 contains the plot of the sum of squared differences from the 128 view reconstruction vs. the number of views. Notice the non-linear aspect to the plot showing the benefit derived from each additional view. This case does not simulate the effect of asymmetries of the center plane. In all the cases, we have placed the asymmetries on the center plane since any single view reconstruction will attribute the asymmetry to the center plane regardless of where the feature is in the 3D object function. Also, we used a cone angle of 7.2 degrees, which will increase the error in the flip operation for the fksingle approach ( the flip of the radiograph is not the 180 view as a function of cone angle). Acquiring extra views will dramatically improve the accuracy of the reconstruction for the case of the general asymmetry. This is precisely the case we have in experimental data. Consequently, these data must be viewed with only casual regard.

Three results have been stated. If the features of interest are at large radii from the center axis, more views will improve the confidence of the reconstruction. If a high level of noise exists in the 
radiography, more views are helpful. If considerable detail exists at random locations throughout the 3D object function, more views are essential. 


\section{Applying CT reconstruction methodology to FXR radiography}

The main reason for developing the techniques discussed in the previous sections of this paper is to aid in the analysis of radiographs taken at the FXR facility, which is located at LLNL's Site 300 firing area. The objects that are radiographed there are usually in the process of exploding at the time of the $x-$ ray pulse, and they are often quite thick, so the pulse must be very short ( $<100 \mathrm{~ns}$ ) and very intense (up to $300 \mathrm{R}$ at one meter). This type of radiography presents us with some unique problems, both in obtaining the data and in analyzing it afterwards, that do not exist in conventional medical or industrial radiography. Of particular significance in the context of this paper is the fact that the number of views is equal to the number of $x$-ray machines that are available (usually only one), since we do not have the luxury of rotating the exploding object and taking a sequence of views.

It will not be possible to discuss in detail here the application of single-view CT techniques to FXR radiographs, since (1)most of the objects we have radiographed are classified, (2)the results tend to be highly object-dependent, and (3)the techniques are still being developed and perfected. Instead, we will discuss those features of an FXR experiment that make it particularly difficult to analyze and will outline the techniques that we are developing to deal with them. Those readers who are interested in more detail should refer to the classified shot reports that will be written for each individual experiment. In general, however, what we have found is that while the use of single-view CT to locate material boundaries in these problems is relatively straightforward, the attempt to use it to obtain quantitative information on mass densities is much more problematic. Further work needs to be done, not only to get answers but also to be able to estimate the uncertainties in those answers.

\section{III.a FXR radiography}

The FXR is a linear induction accelerator which is capable of accelerating a 2.3-kA, 70-ns wide pulse of electrons to an energy of $16.4 \mathrm{MeV}$. These electrons are then focused onto a $1.0-\mathrm{mm}$ thick tantalum conversion target, producing an intense burst of high-energy $\mathrm{x}$-rays. After the $\mathrm{x}$-rays have passed through a 50-cm thick "bullnose" region - which includes some tungsten collimators as well as a vacuum window, a beryllium electron stop, ceramic and beryllium pieces to protect the FXR from blast and shrapnel, and additional shielding materials to reduce the amount of scattered radiation coming from the bullnose - they enter the firing table in the form of a narrow cone beam. Typically, this beam will have a cone angle of $1.0-2.5^{\circ}$ (depending on the particular experiment), an intensity of about $300 \mathrm{R}$ at one meter, and a spectrum which peaks at about $1 \mathrm{MeV}$ and has significant contributions at energies in excess of $10 \mathrm{MeV}$. The effective source spot size is typically $2.2 \mathrm{~mm}$ FWHM, although for one experiment we were able to reduce this to $0.9 \mathrm{~mm}$ by putting additional collimation very close to the target (which also caused a substantial reduction in the intensity). 
The object to be radiographed is placed on the firing table at a distance from the $\mathrm{x}$-ray target which can be as little as $1.3 \mathrm{~m}$ or as much as $9 \mathrm{~m}$, depending on the requirements of the particular experiment. The film is then placed about $50-100 \mathrm{~cm}$ beyond the center of the object. The exact position of the film is usually a compromise between the requirements of (1)minimizing the blur, (2)maximizing the dose at the film, (3)minimizing the scattered radiation at the film, and (4)protecting the film from blast and shrapnel. This last requirement is obviously the most critical one, since we would get no data at all if we destroyed the film. In order to protect the film while still getting it close to the object, we put various types of mitigating material in front of and around the film pack; this material may consist of aluminum, ceramic, and/or dry vermiculite in front, and aluminum or steel around the sides and in back. Unfortunately, this film protection material attenuates the x-ray beam, and it also becomes a significant source of scatter because of its proximity to the film pack.

For many experiments, including the so-called "core punch" shots, we use an additional collimator so as to illuminate only a relatively small region within the object. We do this because the variation in attenuation within the object is so large (ranging from almost no attenuation to as much as a factor of $10^{4}$ or more) that the scattered radiation originating from outside the region of interest could exceed the direct radiation from inside the region of interest. In the case of the core punch, this technique of using very tight collimation is what allows us to look into the very center of a thick imploding object.

Figure 18 shows schematic representations of two typical types of FXR experiments, a core punch and a whole-object shot. Note that for the whole-object shot there is no collimator; the main region of interest is usually at or near the outside surface of the object, and no attempt is made to penetrate the thicker materials in the center. From this point on, we will restrict our discussion to the core punch shots, since those are the ones on which almost all of the interest in CT analysis has been concentrated.

\section{III.b Converting films to exposure and to attenuation}

Whenever the FXR is used to radiograph a reasonably thick object, the $\mathrm{x}$-rays that arrive at the film pack have an energy spectrum which is peaked at about $4 \mathrm{MeV}$, since that is the energy at which the mass attenuation coefficient for high- $\mathrm{Z}$ materials is a minimum. For the same reason, however, those $\mathrm{x}-$ rays are particularly difficult to detect. In a typical FXR experiment, the only way we can get a reasonable detection efficiency (about 30\%) is by stacking a dozen or more films. Each of these films is surrounded by either (a) a pair of high- $Z$ screens which convert some of the $x$-rays into high-energy electrons, which can then expose the film, or (b) the same high- $Z$ screens plus a pair of flourescent screens, which transfer some of the electron energy to visible light photons, which then expose the film. 
This second option produces considerable amplification of the incident $\mathrm{x}$-ray signal and so is essential when the dose is low.

After the films have been recovered and developed, they are digitized with a micro-densitometer. This provides us with a set of computerized images (one for each film) of optical density (OD) vs. position on the film. The first step in the analysis is to convert these OD images into images of relative exposure. For the direct recording films [type (a) above] this is trivial, since the exposure is very nearly proportional to the net OD (i.e., the difference between the actual OD and the fog OD). The value of the fog is determined by developing a piece of unexposed film along with the shot films. The assumptions are that the fog is uniform and that it is the same on all the films: if these assumptions are not correct, then we can make some large errors when measuring small exposures.

If we have to use light-screen films [type (b) above], then the situation is more complicated. In this case, the H\&D curve (i.e., the relation between the OD and the logarithm of the relative exposure) is given by an " $S$ "-shaped curve similar to that used in optical photography. We therefore need to know not only the fog level but also the shape of the H\&D curve. The best way to obtain this is to do a separate experiment, using the FXR, a step wedge, and the same type of film pack as was used on the shot. However, it is more convenient and almost as accurate to simply expose a piece of film to a shortpulse light source through an optical step (or continuous) wedge and then to develop this film along with the shot films. Note that for light-screen films the accuracy is poor at very low exposures, not only because of the uncertainty in the fog level but also because the "gamma" of the film (the derivative of the OD with respect to the log of the exposure) is very low there. Gamma is also low at the highest exposures, where the film is approaching saturation. The best situation for accurate analysis occurs when the OD's are about 1-2 above fog, since gamma is both large and approximately constant within that range.

Once we have the relative exposures, we need to determine the absolute exposures. The ideal way to do this would be to have the darkroom procedures so well controlled and so reproducible that once a calibration of OD vs. absolute exposure had been found for each type of film, the same calibration could be used for all subsequent shots. We have not yet achieved that level of control. In fact, for the direct recording films, there is enough variation in the processing from one batch of films to another that we have to monitor the variation, and correct for it, by developing yet another control film along with the shot films: this one is a film that has been exposed for a known amount of time to a radioactive source. For the light-screen films, fortunately, there is much more consistency, at least on a day-to-day basis (although not yet on a month-to-month or year-to-year basis).

The way we get around our lack of knowledge of the absolute exposures is to use a radiographic mock. The mock is a static object which is designed to have approximately the same range of transmissions and the same mix of high- $\mathrm{Z}$ and low- $\mathrm{Z}$ materials as the dynamic object. Since we can calculate the transmissions through the mock, and since we monitor the dose coming out of the FXR on 
each pulse, and since we use the same film pack developed in the same way for both the mock and the shot, we can therefore use the mock data to normalize the shot data and to convert them to absolute transmissions through the dynamic object. This is what we have done on each of our core punch experiments to date.

There is one further difficulty in going from OD to object transmission, and that has to do with the removal of scatter. Scattered radiation is a very serious problem in high-energy flash radiography, and we expend considerable effort both to minimize it and to calculate it accurately. When we refer to "scatter", by the way, we mean not only Rayleigh and Compton scattering, but also secondary bremsstrahlung and annihilation radiation, neither of which is significant in low-energy radiography. The scatter originates primarily from (1)the region surrounding the $x$-ray source, (2)the object, (3)the film protection materials, and (4)the film pack itself. We minimize it mostly by using a large amount of shielding around the source region, by using light, thin materials for the film protection (generally a few centimeters of 7075-T6 aluminum alloy), and, most importantly, by heavily collimating the incident $\mathrm{x}$ ray beam so that it illuminates only those parts of the object that the designer is really interested in.

However, since there is always some scatter, and since it is sometimes comparable to or even greater than the direct radiation in some parts of the image, it is very important to remove it from the data. This can be done in part by looking at those regions of the film that are in the shadow of the collimator, where there should be little or no direct radiation, and assuming that the exposure there is entirely due to scatter. The trick then is to extrapolate from there into the region of interest: to do that it is necessary to use a theoretical calculation of the scattering. Single-scattering approximations are usually not adequate for this purpose because of the thickness of the object, so Monte Carlo calculations are needed. Unfortunately, these calculations require a knowledge of the detailed structure of the object, which of course is the very thing we are trying to determine, so some iteration may be necessary.

\section{III.c Using multiple films}

In a core punch experiment, there is always a problem with noise. This is predominantly shot noise, which is a result of the limited number of detected x-ray photons. In order to reduce the noise, it is necessary to increase the detection efficiency, which we do by stacking a dozen or more films in the film pack. If the distance between the films is greater than or equal to the mean electron range, then the

signals on the different films are statistically independent, and we can substantially increase the signal to noise ratio (SNR) by combining the films. This is usually done on the computer, either before or after the conversion from $O D$ to exposure:

Care must be taken to register the images properly before adding them, so as not to degrade the resolution of the combined image. Also, since we normally put at least three different film/screen combinations in the film pack, there are some difficulties involved in adding them together. For one 
thing, the faster films have worse resolution: in particular, the light-screen films are substantially worse than the direct recording films. Therefore, if we simply added those two types together we could lose the relatively good edge resolution which exists on the direct recording films (Of course, even for the direct recording films, the resolution is worse than it is in low-energy radiography, because the highenergy electrons generated in the screens can travel sideways by as much as a millimeter before they expose the film). Also, the low-gamma portions of the image on the light-screen films have a low SNR (when the signal is expressed in units of exposure), so adding these to the higher-gamma portions of the other films could actually make the combined SNR worse.

An example of the improvement in SNR that can result from combining films is shown in Figure 19.

\section{III.d CT reconstruction of EXR radiographs}

Once we have analyzed the radiographic data and expressed them as transmissions through the object, we ought to be ready to do a CT reconstruction using the techniques outlined in Section II of this paper. However, there is one more step that is required, and it is one that is not typically needed in conventional CT. Recall that core punch radiography can be done only if there is severe collimation of the incident $\mathrm{x}$-ray beam. If the entire object were illuminated, as in conventional CT, the resulting scatter would overwhelm the signal in the region of greatest interest. Thus the price for getting a goodquality image of the center of the object turns out to be the loss of information that is vital for the reconstruction of the object.

There are ways to overcome this difficulty, but they all involve the use of assumptions about those parts of the object that are outside of the collimated field of view. The simplest assumption, as illustrated in Figure 20(a), is that the transmission through these "background" regions is a constant. Thus, if we define a sub-object or "core" region whose boundary lies just inside the collimator boundary, the constant background transmission can be determined from the data between the core and the collimator. We can then divide the experimental transmissions by the background transmission, and what we are left with is an image of the transmissions through the core alone, as if it were an object in its own right, suspended in mid-air. The core is then amenable to single-view CT analysis, as described above. Unfortunately, this technique is applicable only in very specific circumstances, which do not usually occur in LLNL core punches.

A better assumption, illustrated in Figure 20(b), is that, although the transmission through the background regions is not a constant, it can be determined to an adequate level of accuracy by using a hydrocode calculation. Then within the core region the experimental transmission for each pixel of the image can be divided by the calculated background transmission for that pixel, resulting again in an inferred transmission through the core. This technique can work well if there is high confidence in the 
hydrocode calculation, if there is not too much structure in the calculated background, and if the background transmission is large compared to the average core transmission. Of course, if any of these conditions does not apply, the CT reconstruction of the core could be seriously in error.

Another complication arises from the phenomenon of spectral hardening. That is, as the $\mathrm{x}$-ray beam passes through the object (and through the collimator and the film protection) its energy spectrum continuously changes due to the strong energy dependence of the mass attenuation coefficients. Therefore, all of the calculations of the transmissions through the background regions, the collimator, etc., must be done with the proper spectral weighting. Furthermore, the transmission through any part of the system depends on the spectrum that results from the transport of the $x$-rays through all the previous parts of the system. It is therefore very important to do the "bookkeeping" correctly.

Another consequence of spectral hardening arises after the CT reconstruction has been completed. What the reconstruction provides is the linear attenuation coefficient in each voxel (expressed in $\mathrm{cm}^{-1}$ ), which has to be divided by the appropriate mass attenuation coefficient (expressed in $\left.\mathrm{cm}^{2} / \mathrm{g}\right)$ so that we can get the mass density $\left(\mathrm{g} / \mathrm{cm}^{3}\right)$, which is what we really want. The difficulty arises when we try to determine the appropriate mass attenuation coefficient. This will depend on the identity of the material in that particular voxel, and it will also depend on the amount of spectral hardening that the $x$-rays have experienced by the time they arrive there. So even if everything has been done correctly all the way from the raw data through the CT analysis, there will still be some uncertainty in the final answer unless we deal correctly with this effect. 


\section{Summary and Discussion}

The first part of this investigation focused on the ability to perform CT reconstuctions from small numbers of radiographic views (1-2), and the second part applied these techniques to FXR radiography of dynamic events. In the first part of the investigation simulated data was used as the best means of assessing the quantitative accuracy of CT reconstructions from single radiographic views. Since our interest is in applying these techniques to acquired data, the performance of the algorithms when assumptions were only true to some degree was of acute interest. A set of simulations and reconstructions was performed to assess the impact of: i) high contrast asymmetries, ii) low contrast asymmetries, iii) errors in the specification of the source-detector geometry, and iv) overwhelming noise. A number of conclusions can be advanced from this evaluation of different conditions applied to the simulated data for single view CT reconstructions.

i) High contrast asymmetries generate the most problems for single-view CT reconstructions. The effects are worse as the feature is at a larger radius from the center axis of symmetry, and when the amount of contrast is greater.

ii) Low signal to noise, or high noise, in the radiograph will overwhelm single view $C T$, since the reconstruction operation, which unfolds the path length, determining the per-voxel amount of attenuation from the comparatively large ray-sum integral, is inherently noise amplifying. Typically, without additional smoothing, the noise amplification is a factor of between 10 to 30 on the center pixel [WINER], and possibly higher depending upon the particulars of the algorithm. However, while smoothing the input radiograph can reduce the noise level, a direct loss in spatial resolution will result.

iii) Knowlege of source-detector geometry is important, and neglect of the fan/cone angle will exacerbate other inaccuracies.

iv) The 'fksingle' approach to obtaining the un-acquired views performs reasonably well in the presence of small amounts of asymmetry, or of spatially diffuse, low-contrast asymmetries.

v) Single-view reconstructions can provide locally accurate spatial distributions of internal features even in the presence of a certain amount of asymmetry. Quantitative accuracy will be affected by the departures in assumptions in the input data, and by the accuracy of the transmission radiograph itself. 
The results of the simulations provide justification for using single-view CT techniques to obtain qualitative images of the internals of dynamic events. Boundaries and spatial properties are correctly identified in spite of limited problems with assumptions. All departures from assumptions do affect the quality of the image, but impact the quantitative properties of the image first, allowing the experimenter to obtain a view of boundaries in spite of limited inaccuracies. Computational procedures can be applied to remedy some of these problems but not without other consequences.

When we attempt to apply these techniques to FXR flash radiographs, we encounter a whole new set of problems, in addition to those that are inherent in single-view CT. First, considerable effort must be expended in preparing the radiographs for the CT analysis. The films must be digitized, the digitized files must be added together in an appropriate way to form a combined image, the conversion from optical density to relative exposure must be done correctly, spectral hardening must be properly accounted for, and the mock image (which was obtained using the same techniques) must be used to properly normalize the exposures and produce an image of transmissions through the object.

Secondly, we have to deal with the problem of scatter. Scattered radiation is ubiquitous in highenergy radiography, and, although various techniques have been developed to minimize it, it cannot be eliminated or even made negligibly small. Instead, calculational techniques have to be used to estimate the magnitude and distribution of the scattered component of the image, so that it can be removed.

Thirdly, there are problems with noise and blur. The noise is the result of the limited number of $\mathrm{x}$-ray photons that are detected. The blur is the result of (a) the relatively large source spot size and (b) the sideways spreading of the high-energy electrons in the film pack. Both the noise and the blur are worse than they are for the typical low-energy CT measurement.

Finally, we have to deal with the fact that only part of the object (perhaps a very small part) is imaged on the film, as a result of the collimation that was used to minimize the scatter. Some assumptions and calculations are needed to account for the "'background" materials, i.e., the materials that are outside the field of view but still contribute to the total transmission through the object.

Despite all these problems, we have already had some success in using single-view CT to obtain qualitative results from FXR radiography, including such important information as the location of material boundaries and shock fronts. Having the capability of displaying the data as material distributions inside the object rather than as projections through the object is very helpful to those who need to interpret the results of the experiment. Further work is proceeding on the much more difficult problem of extracting reliable quantitative information from the radiograph. The ultimate goal is to get an accurate image of the absolute material densities in the center of the object, but we still have a considerable way to go before we achieve that goal. 


\section{Figure Captions}

Figure 1 - CT reconstructions illustrating the effects of an incorrect center of rotation in the reconstruction.

Figure 2 - Illustration of the geometry and coordinates for the CT reconstruction fundamentals.

Figure 3 - Images of the base high contrast asymmetries and low contrast asymmetries used in the simulations.

Figure 4 - Different cases used in the simulations for the high contrast asymmetries

Figure 5 - Different cases used in the simulations for the low contrast asymmetries

Figure 6 - Simulation used for evaluating errors in source-detector geometry .

Figure 7 - Plot of the reconstruction errors from the 'fksymm' and the 'fksingle' codes for the high contrast asymmetries

Figure 8 - Images showing the localized character of the error in the 'fksymm' reconstructions in the presence of high contrast asymmetries.

Figure 9 - Images showing the localized character of the error in the 'fksingle' reconstructions in the presence of high contrast asymmetries.

Figure 10 - Plots of maximum absolute difference for the 'fksymm' and 'fksingle' approaches to reconstructing in the presence of the high contrast asymmetries.

Figure 11 - Plots of the 'fksymm' and the 'fksingle' results for the low contrast data.

Figure 12 - Plots of the total reconstruction error and the maximum error as correlated with fan/cone angle

Figure 13 - Images of a parallel reconstruction, cone beam reconstruction, and the difference showing the localized character of the errors for 3 degree cone angle data.

Figure 14 - Different single view reconstructions for varied levels of input noise

Figure 15 - Image showing the propagation of noise in an LLNL written Abel Transform code

Figure 16 - Noise to signal in the reconstructed image for different levels of noise to signal in the input radiograph.

Figure 17 - Reconstruction error for reconstructions with additional numbers of views

Figure 18 - Schematic representations (not to scale) of two typical types of FXR radiographic experiments: (a) a core punch, in which only the center of the object is imaged; (b) a whole-object shot, in which the entire object is imaged. 
Figure 19 - Comparison of single film and multiple film (summed) images from FXR radiography

Figure 20 - Approximate methods for dealing with materials outside the collimated field of view ("background"): (a) assuming the background is uniform; (b) assuming the background is described accurately by a hydrocode calculation. 


\section{References \& Bibliography}

[ASTM] 'Standard Guide for Computed Tomography (CT) Imaging', Version 3.1 ASTM , Dr. Jim Stanley

[ASNT85] Nondestructive Testing Handbook, Volume III Radiography \& Radiation Testing, Bryant L. and P. McIntyre, Part 2 Principles of Gamma and X-ray attenuation gaging , pps. 678-681, American Society for Nondestructive Testing, 1985

[MARTZ89] "Design, Performance, and Application of a CCD camera-based CT system", H.E. Martz, M.F. Skeate, D.J. Schneberk, S.G. Azevedo, Proceedings of the ASNT CT Topical conference, Seattle, WA, June 1989.

[MARTZ90a] 'Computerized Tomography', H.E. Martz G.P. Roberson, D.J. Schneberk, and S.G. Azevedo, November-December 1990 issue of Energy and Technology Review (UCRL-52000-90-1112), Lawrence Livermore National Laboratory, Livermore, California.

[MARTZ90b] H. E. Martz, S.G. Azevedo, J.M. Brase, K.E. Waltjen and D.J. Schneberk, Computerized Tomography "Computerized Tomography Systems and Their industrial Applications," Appl Radiat. Isot. 41:943, (1990).'

[MARTZ91] H.E. Martz, G.P. Roberson, D.J. Schneberk, and S.G. Azevedo, "Nuclear-SpectroscopyBased, First-Generation, Computerized Tomography Scanners" IEEE Trans. Nucl. Sci. 38:623, (1991).

[SCHNE90a] 'tport - a code for calculating x-ray attenuation properties of arbitrarily complicated compounds', private communication.

[SCHNE90b] "Sources of Error in Industrial Tomographic Reconstructions," D. J. Schneberk, S. G. Azevedo, H. E. Martz, and M. F. Skeate, Materials Evaluation, Columbus, Ohio, 48:609 (1990).

[SCHNE91] "Multiple-Energy Techniques in Industrial Computed Tomography,", D.J. Schneberk, H.E. Martz, and S.G. Azevedo, Review of Progress in Quantitative Nondestructive Evaluation, D. O. Thompson and D. E. Chimenti, Eds. (Plenum Press, New York, 1991) pp 451-458.

[AZEVEDO90] "Calculation of the Rotational Centers in Computed Tomography Sinograms,", S.G. Azevedo, D.J. Schneberk, J. P. Fitch, and H.E. Martz, IEEE Trans. Nucl. Sci. 37:1525 (1990)

[AZEVEDO91a] "Estimation of Geometrical Parameters in Parallel-beam, Fan-beam, and Cone-beam CT Systems,", S.G. Azevedo, D.J. Schneberk, J. P. Fitch, H.E. Martz, M. F. Skeate, and G.P. Roberson, Review of Progress in Quantitative Nondestructive Evaluation, D. O. Thompson and D. E. Chimenti, Eds. (Plenum Press, New York, 1991) pp 459-468.

[AZEVEDO91b] "A comparison of Feldkamp and Radon Cone-Beam Reconstructions,", S.G. Azevedo, LETI, DSYS/SETIA/030991/C325, September 1991

[AZEVEDO92] "Region of Interest Tomography with the RADON reconstruction Algorithm,", S.G. Azevedo, P. Rizo, P. Grangeat. Review of Progress in Quantitative Nondestructive Evaluation, D. O. Thompson and D. E. Chimenti, Eds. (Plenum Press, New York, 1991) pp 459-468.

[JOHNS82] "Scattered radiation in fan beam imaging systems", P. C. Johns, M. Yaffe, Medical Physics, Vol. 9, No. 2, March/April 1982. 
[SEGAL90] "Computerized Laminagraphy", Y. Segal and B. Cohen, NDT International, June 1990.

[BARRETT81], Barrett H.H., and Swindell W.,. Radioliogical Imaging - The theory of Image Formation, Detection and Processing, Vols I \& II, Academic Press, New York (1981).

[KAK87] A. C. Kak, and M. Slaney, Principles of Computerized Tomographic Imaging, New York: IEEE Press, 1987.

[KOURIS82] K. Kouris, N. M. Spyrou, and D. F. Jackson, in Research Techniques in Nondestructive Testing, Vol VI, edited by R. S. Sharpe (Academic Press, New York 1982)

[GRANGEAT87] P. Grangeat, "Analyse d'un systeme d'Imagerie 3D par Reconstruction a partir de Radiographies X en Geometrie Conique". Phd Thesis (1987)

[GRANGEAT88] P. Grangeat, "3D Image Reconstruction in Non-Destructive Testing", CEA - D.LETI Electronics Acquisition Systems Department, March 1988.

[HANSON82] K. M. Hanson, "CT reconstruction from limited projection angles," Proc. SPIE (Appl. Opt. Instr. Med. X), Vol. 347, pp. 166-171, 1982.

[DINTON90] J. M. Dinten, "Tomographic Reconstruction of Axially Symmetric Objects: Regularization by a Markovian Modelization," Proc. of the Tenth International Conference on Pattern Recognition, Atlantic City, New Jersey, June 16-21, 1990, IEEE Computer Society Reprint, Los Alamitos, CA

[WINER], Kris Winer and D. J. Schneberk, "An analysis of Noise Amplification in limited view CT (1 4 views)", LLNL internal memorandum, February 1995.

[LANSFORD] C. A. Lansford, "Limited view CT of Uranium Fuel Tubes", LASL report, 1988.

[HARMS77] A. A. Harms, and A. Zeilinger, "A New Formulation of Total Unsharpness in Radiography", Phys. Med. Biol., 1977, Vol. 22, No. 1, 70-80.

[LOGAN92] C.L. Logan "New Methods for Mammography" (to be published).

[SEIBERT84] J. A. Seibert, O. Nalcioglu, W. W. Roeck, "Characterization of the veiling glare PSF in $x-$ ray image intensified fluoroscopy", Med. Phys. 11(2), Mar/April 1984.

[SEIBERT85] J. A. Seibert, O. Nalcioglu, W. W. Roeck, "Removal of image intensifier veiling glare by mathematical deconvolution techniques, Med. Phys. 12(3), May/June 1985.

[SEIBERT88] J. A. Seibert, J. M. Boone, "X-ray scatter removal by deconvolution ", Med. Phys. 15(4), Jul/Aug 1988.

[BOONE88] J. M. Boone, J. A. Seibert, "An analytical model of the scattered radiation distribution in diagnostic radiology", Med. Phys. 15(5), Sep/Oct 1985.

[PLA91] R. Placious, H. Berger, J. Rogerson, and C. Bueno, "A High Performance Scintiallator for Real Time X-ray Applications", Materials Evaluation, December 1992. 


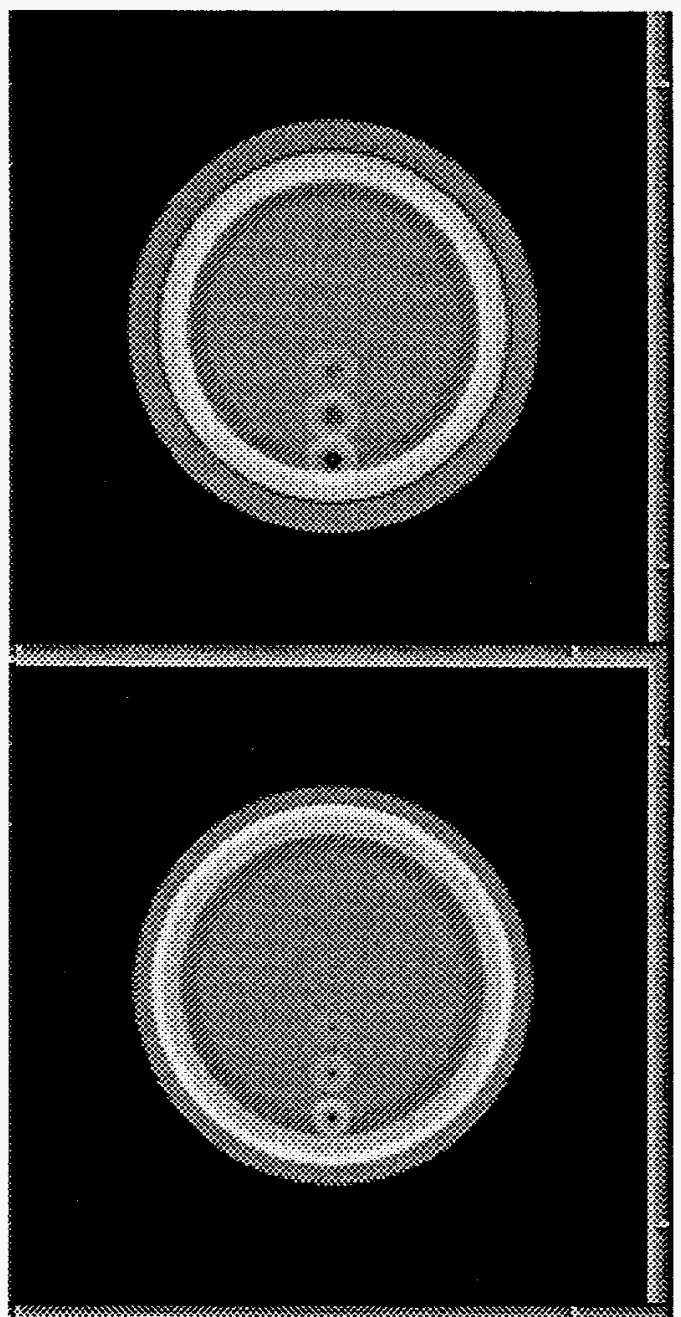

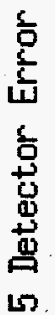

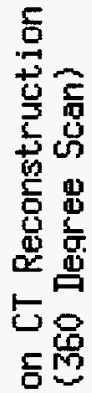

客

号高

营 方

M d

$+$

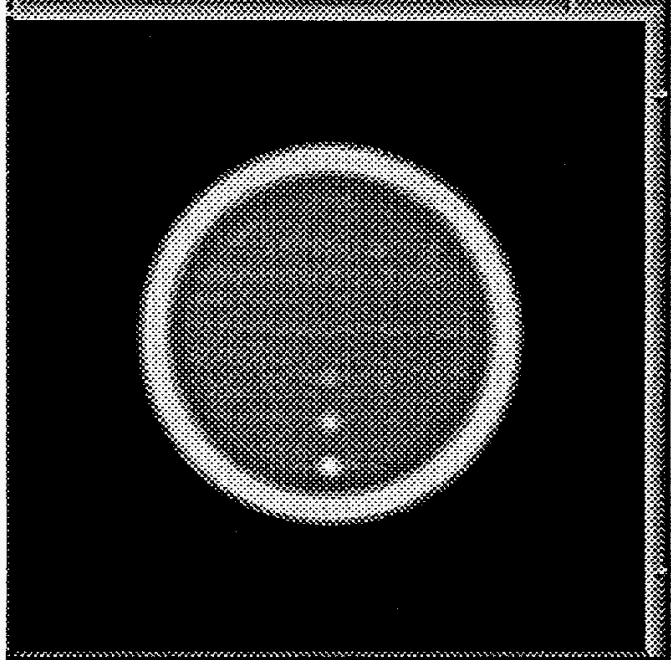

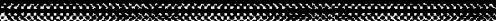

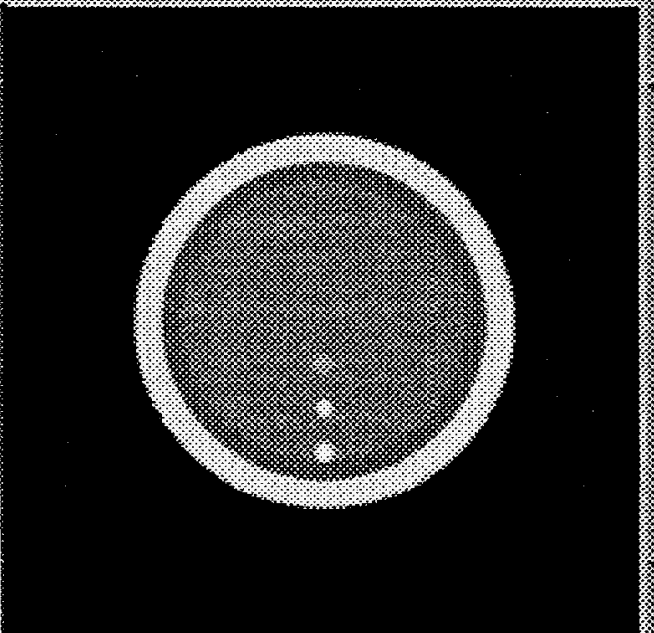

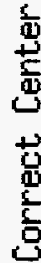



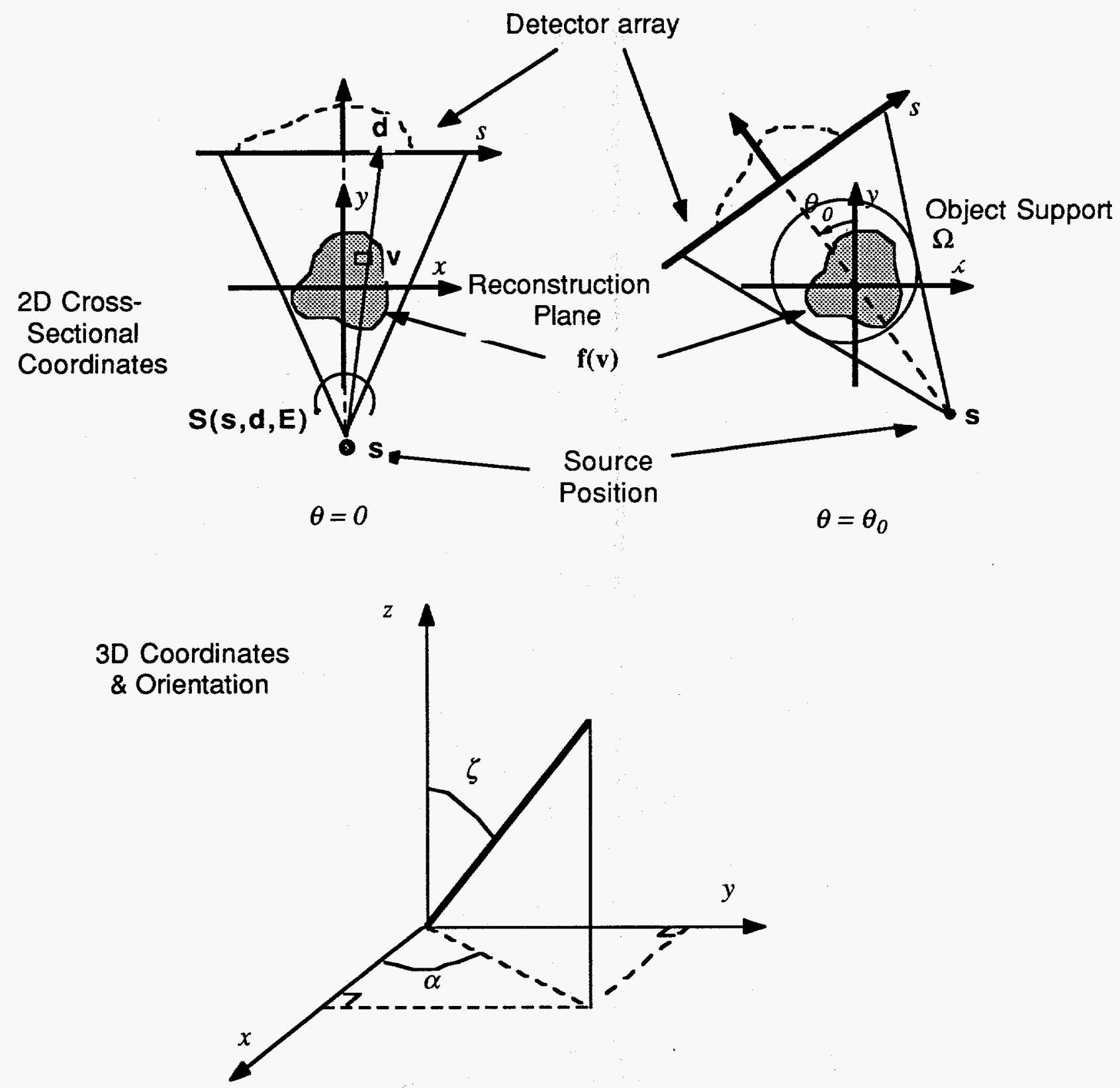

Figure 2 - Geometry for CT Reconstructed Data 


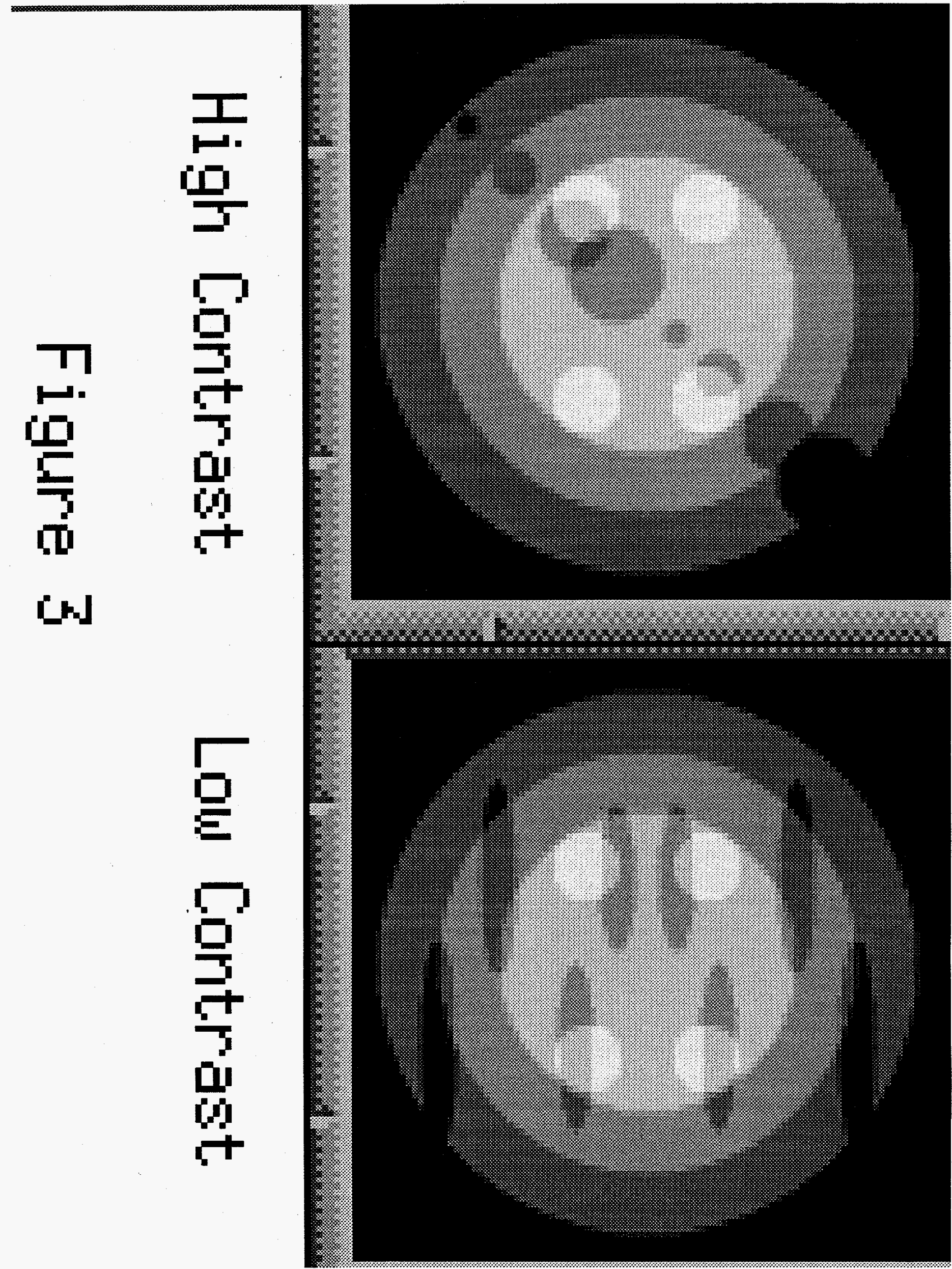




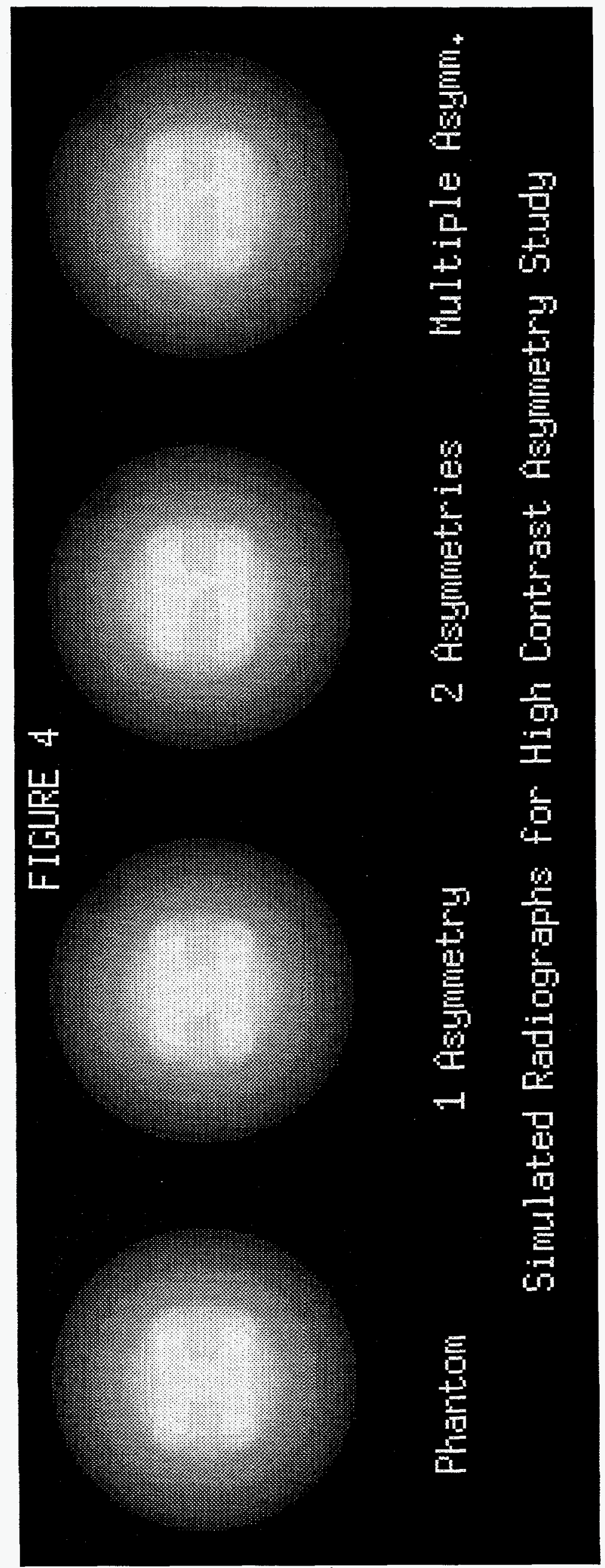




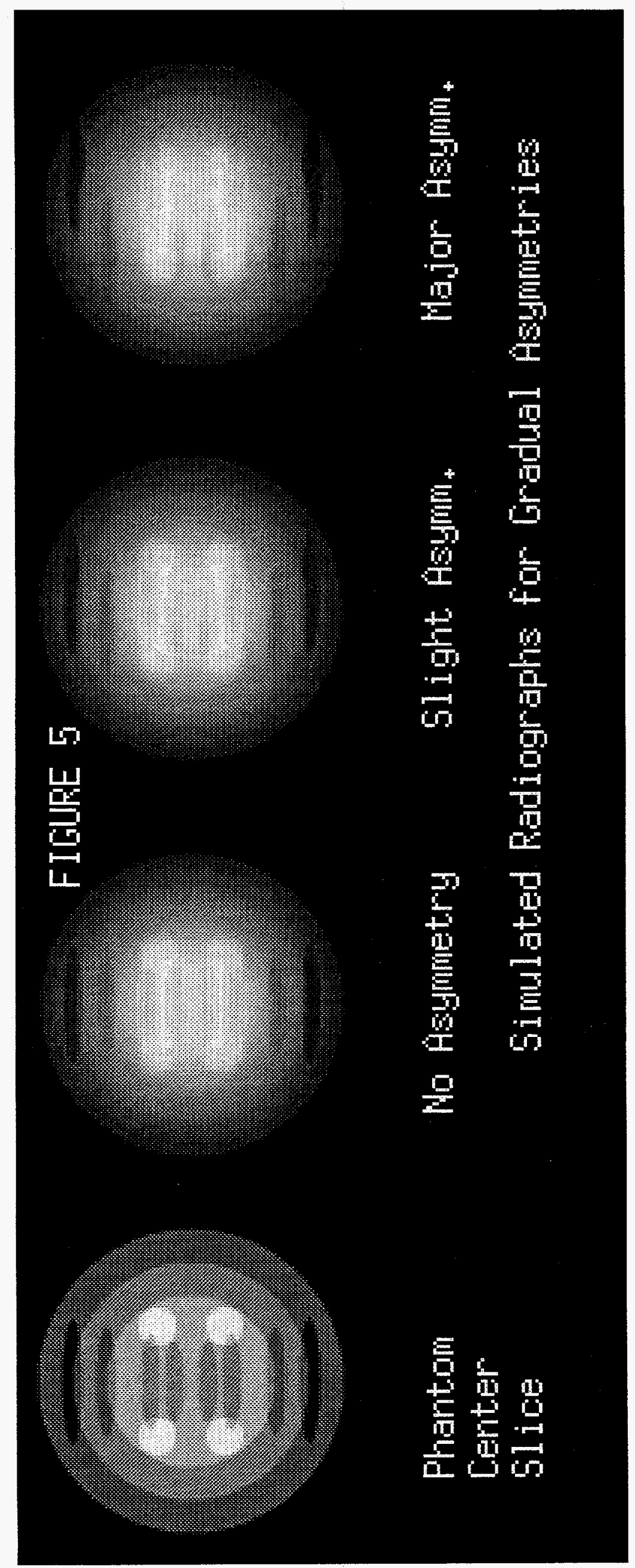




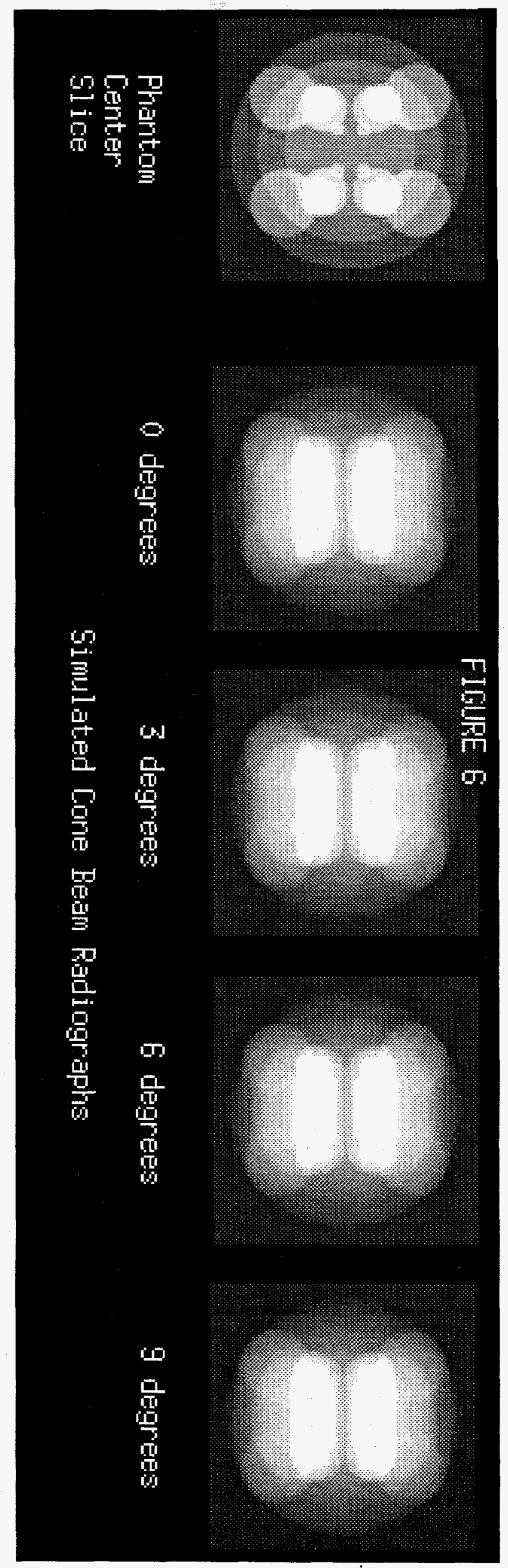




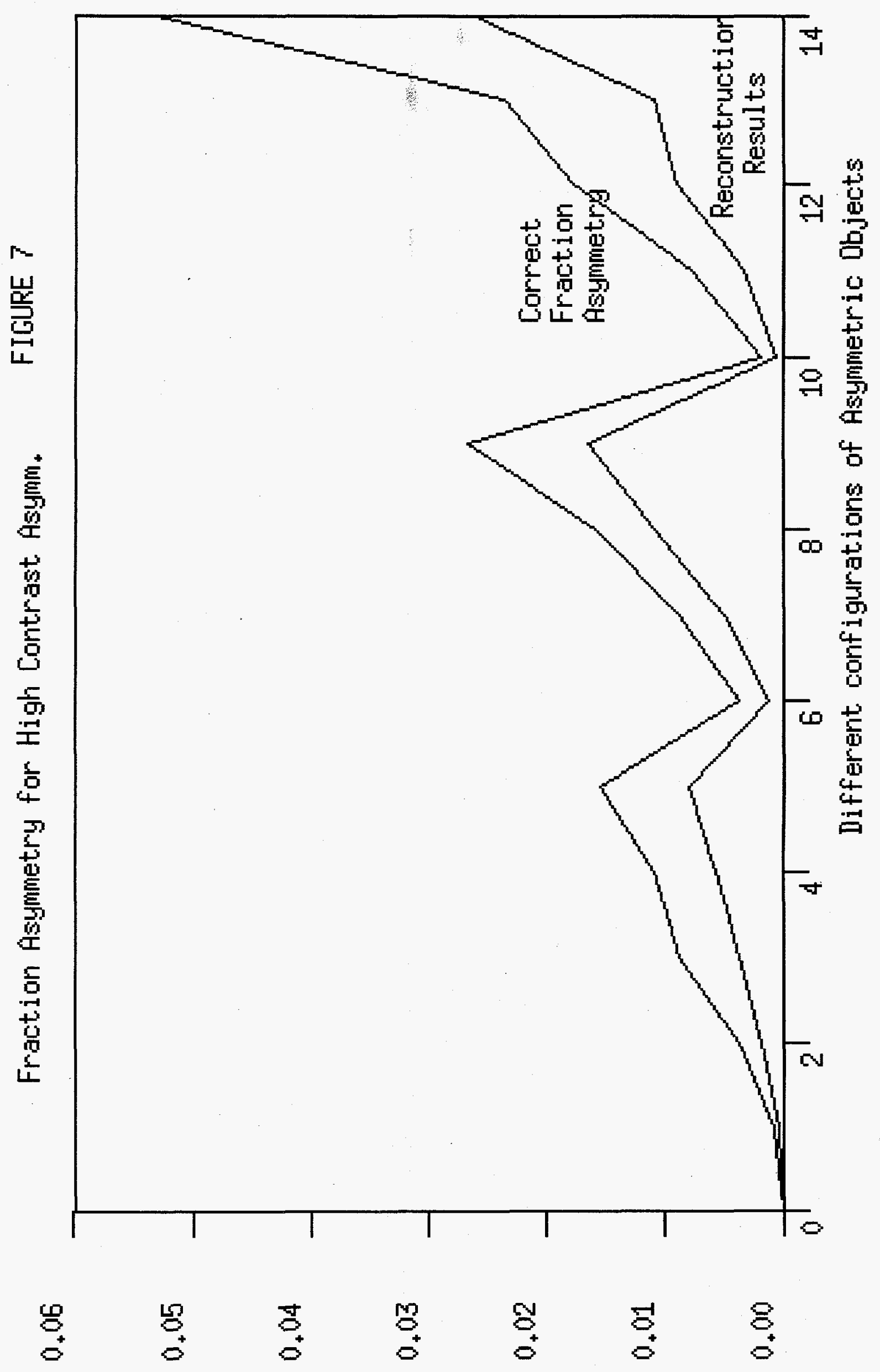




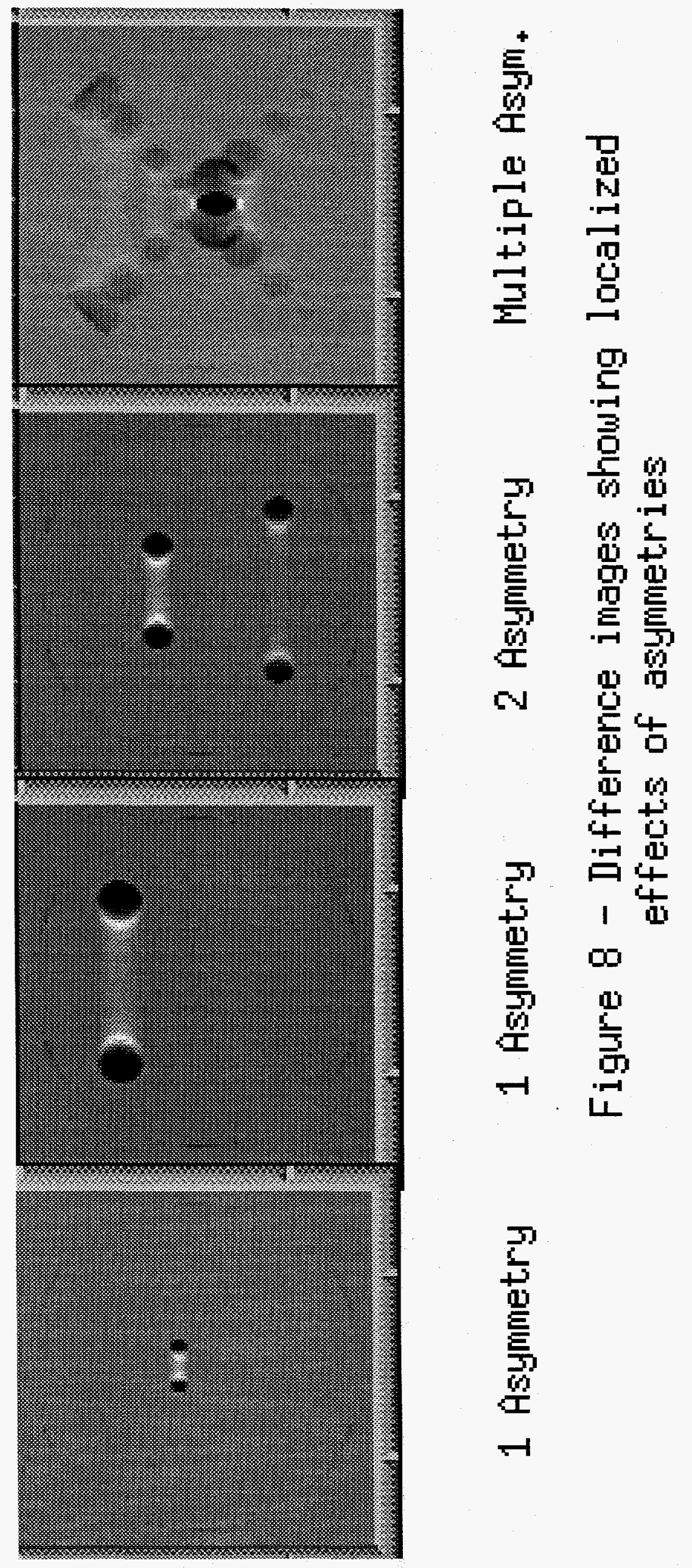




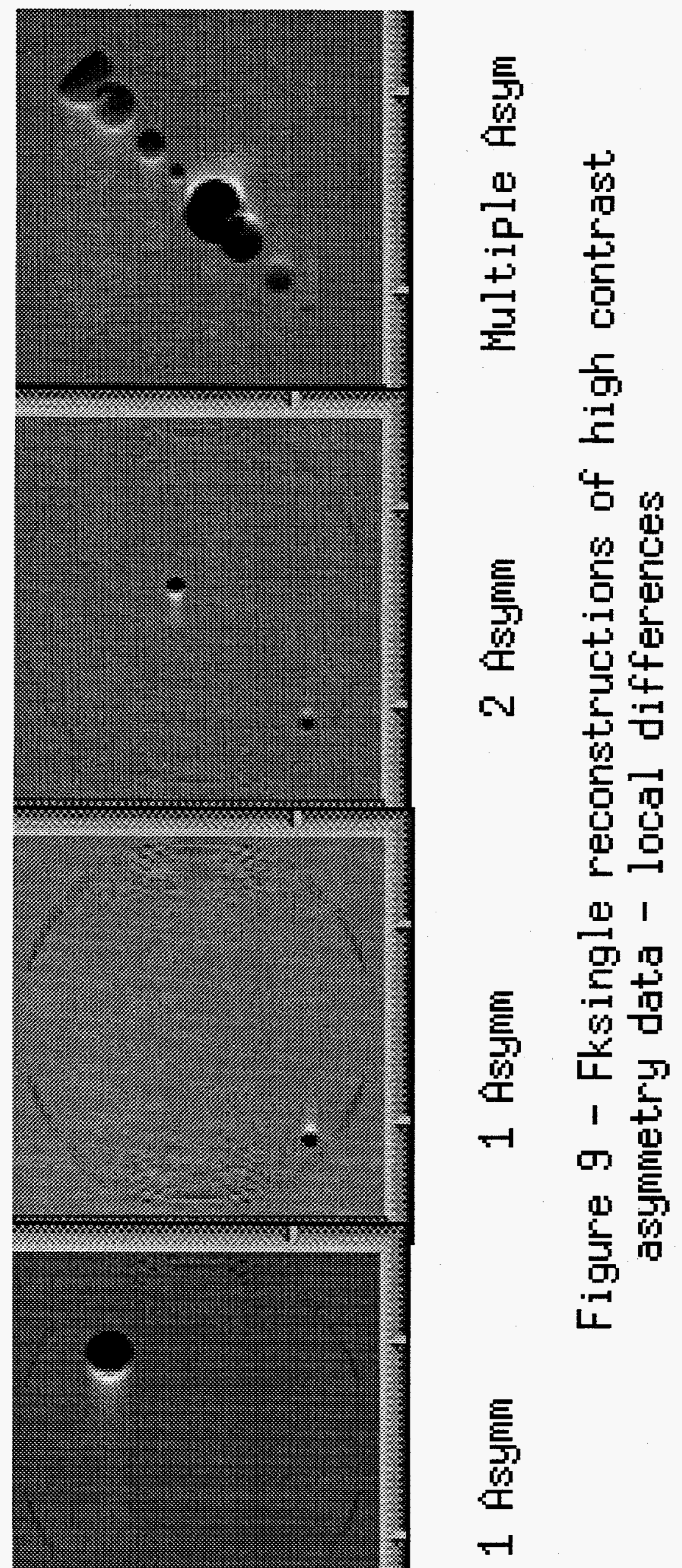




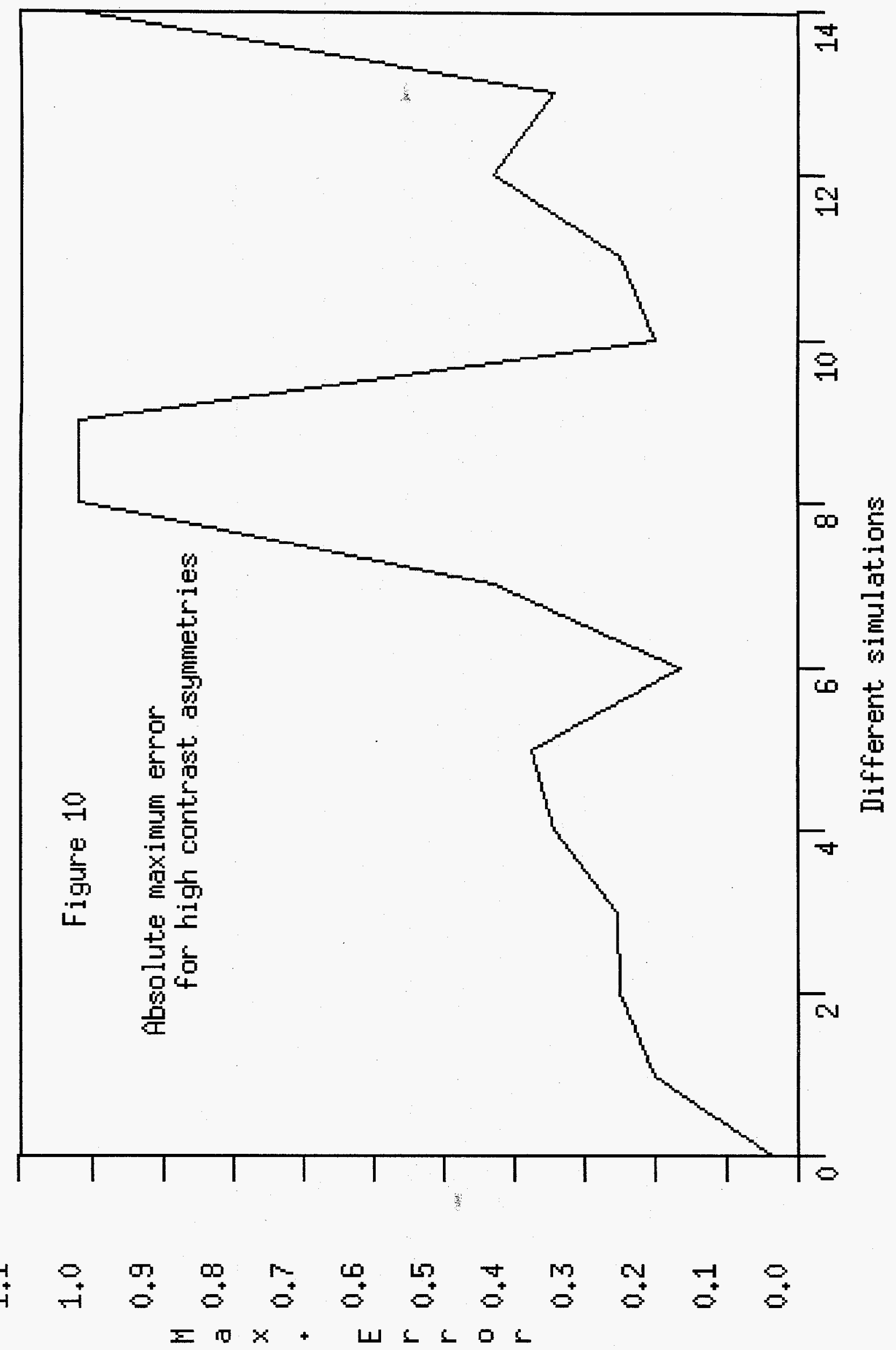




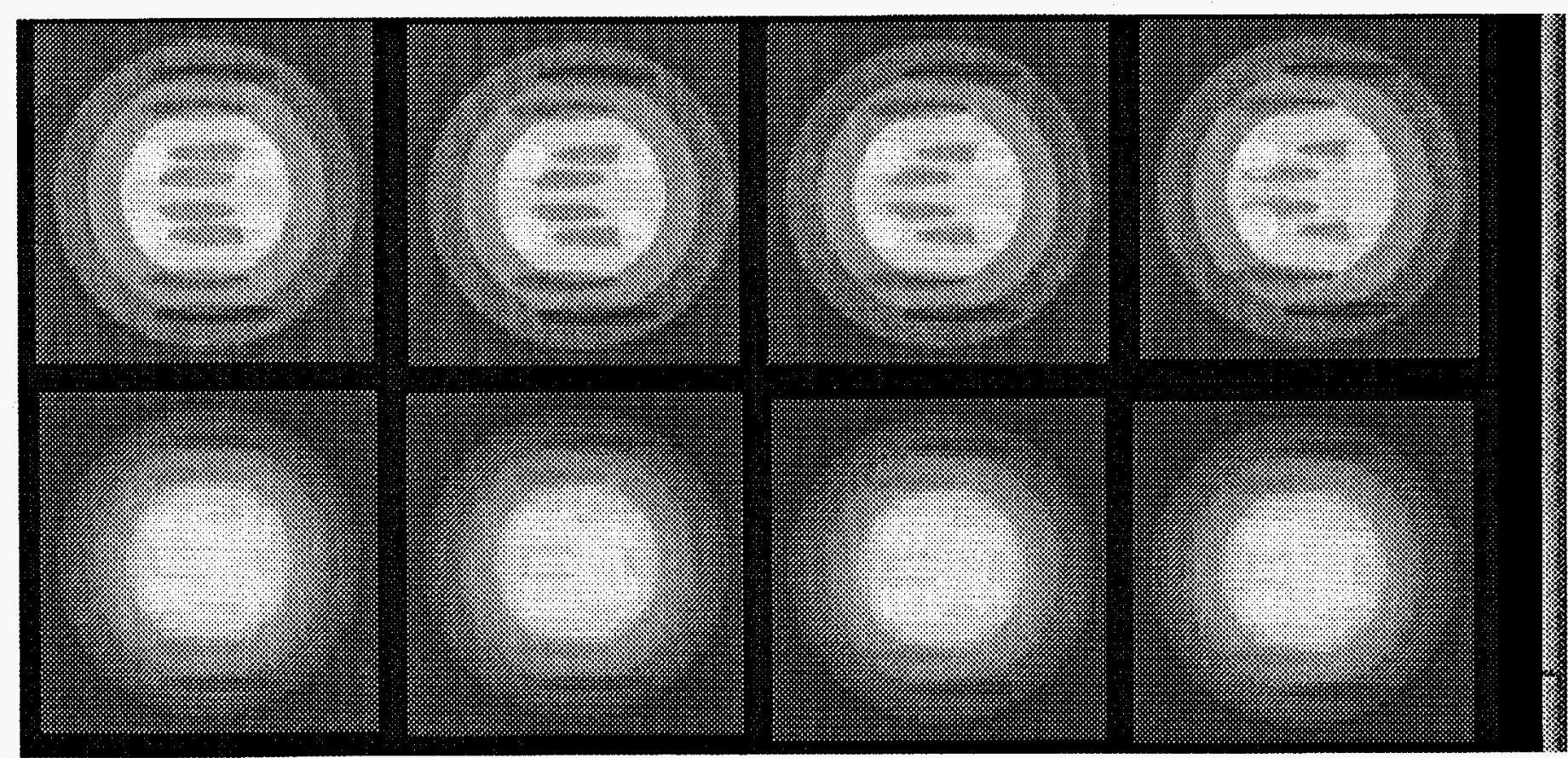

Recontructed Ilata

using fksingle

Simulated Radiographs

Figure 11 - Low contrast 


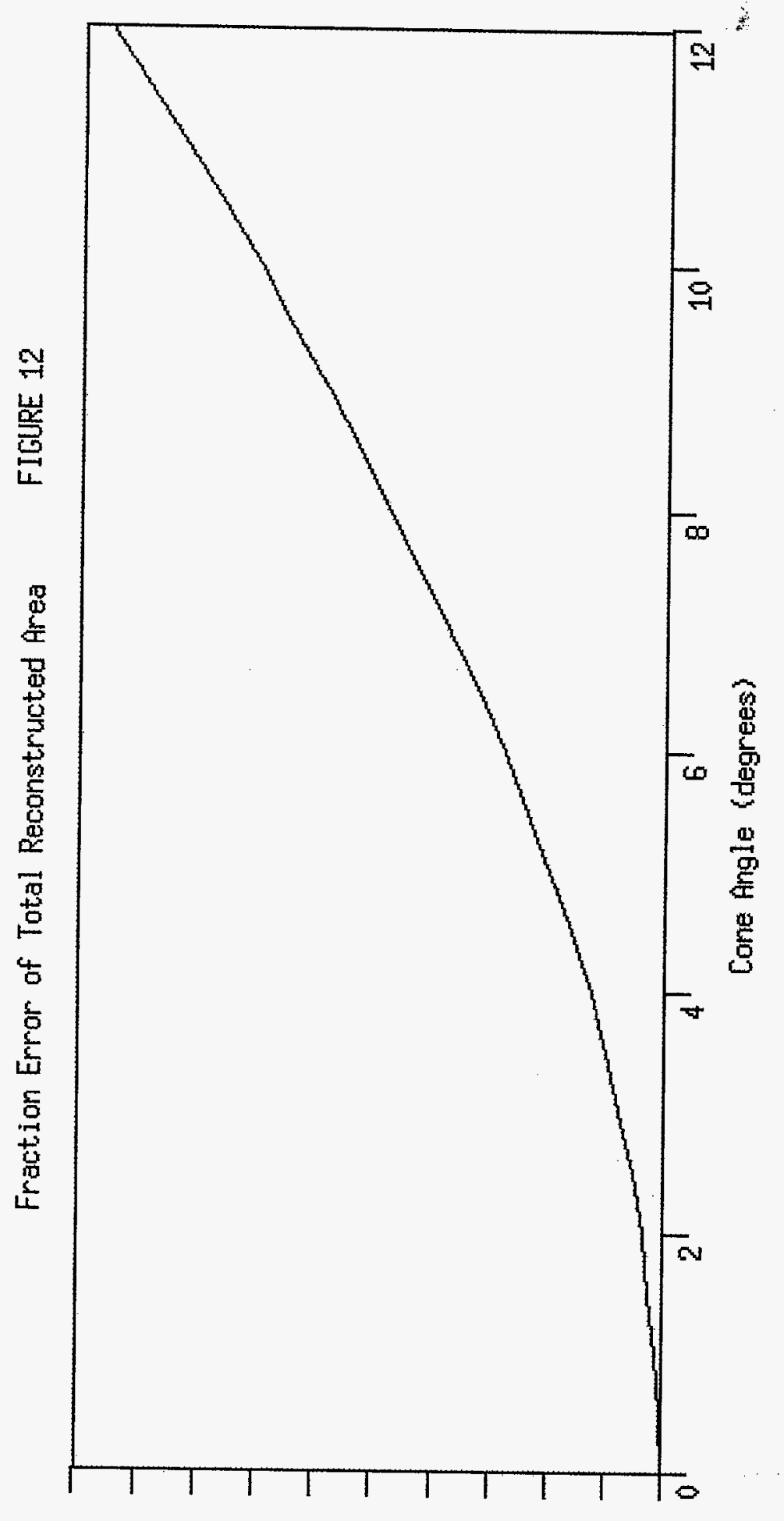

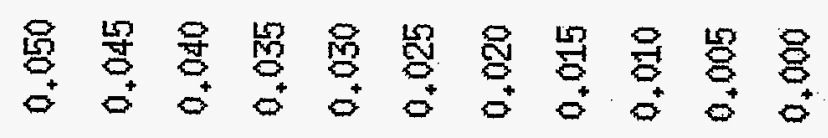

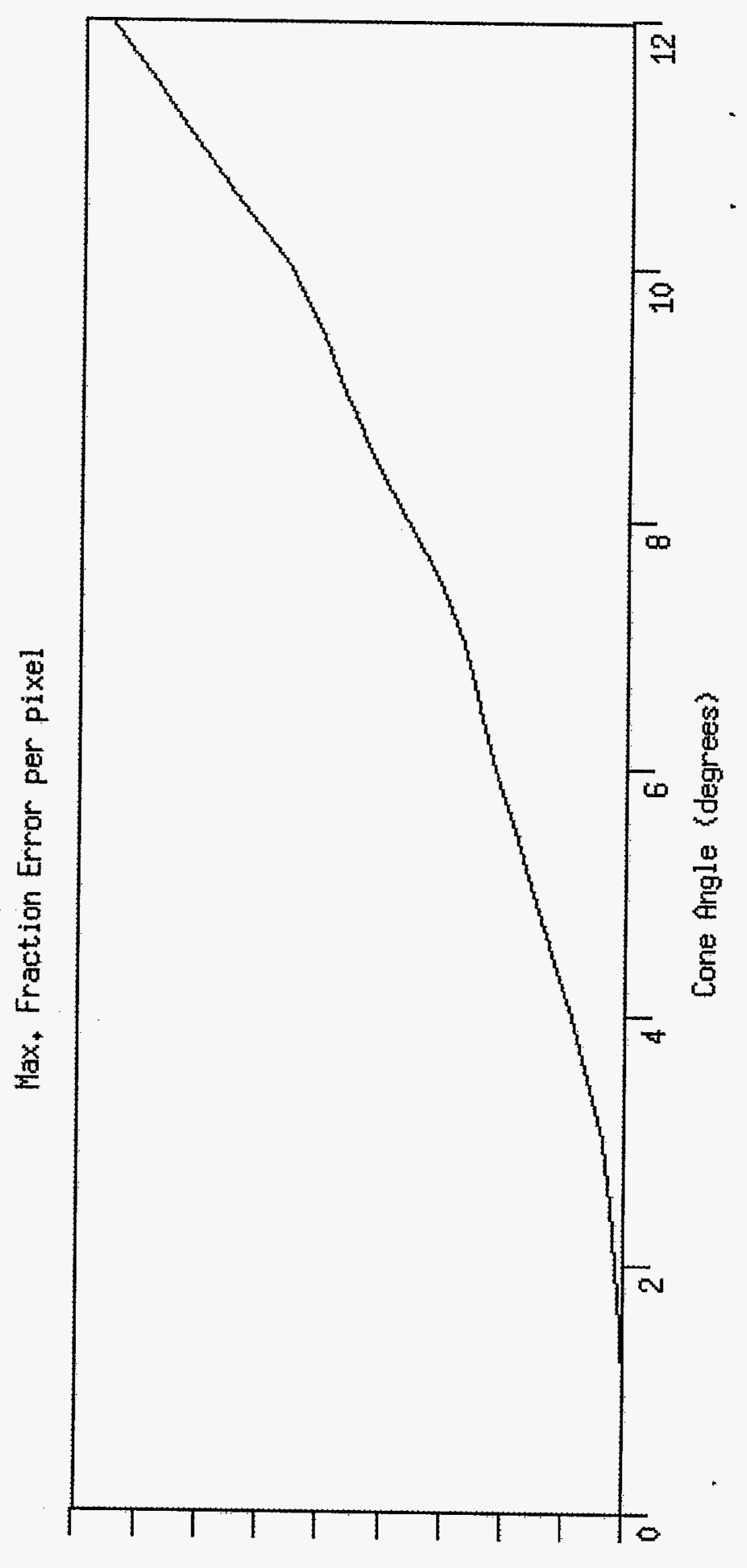

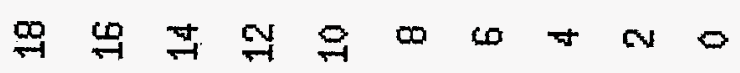




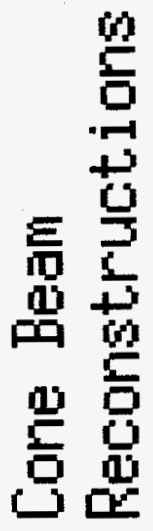
$=\frac{5}{0}=$
(1)
$\rightarrow \frac{3}{2}$
음
$\rightarrow$ in
品
iD
5 $01+$
10
4 믄
뭄무
4
놈

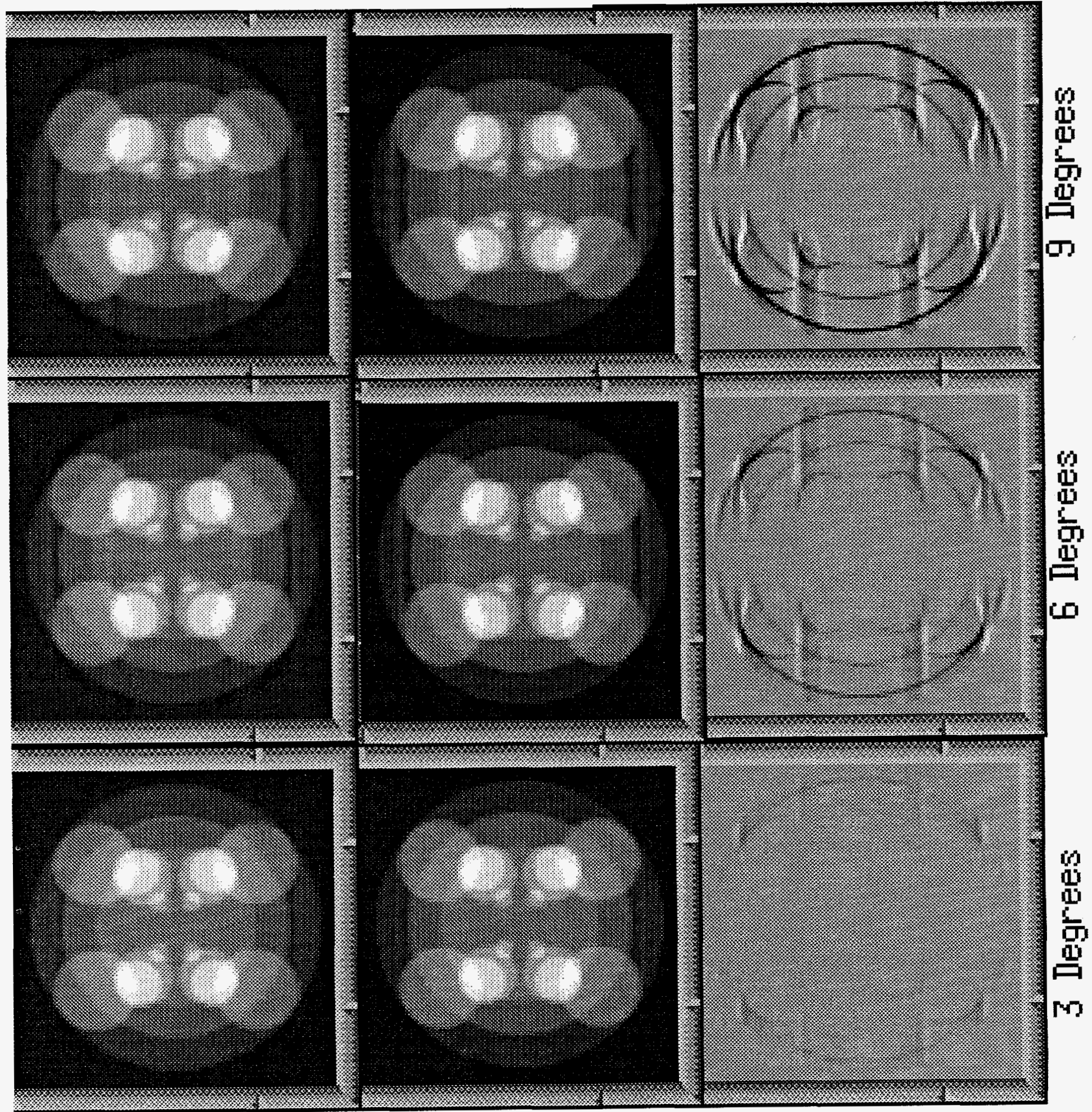




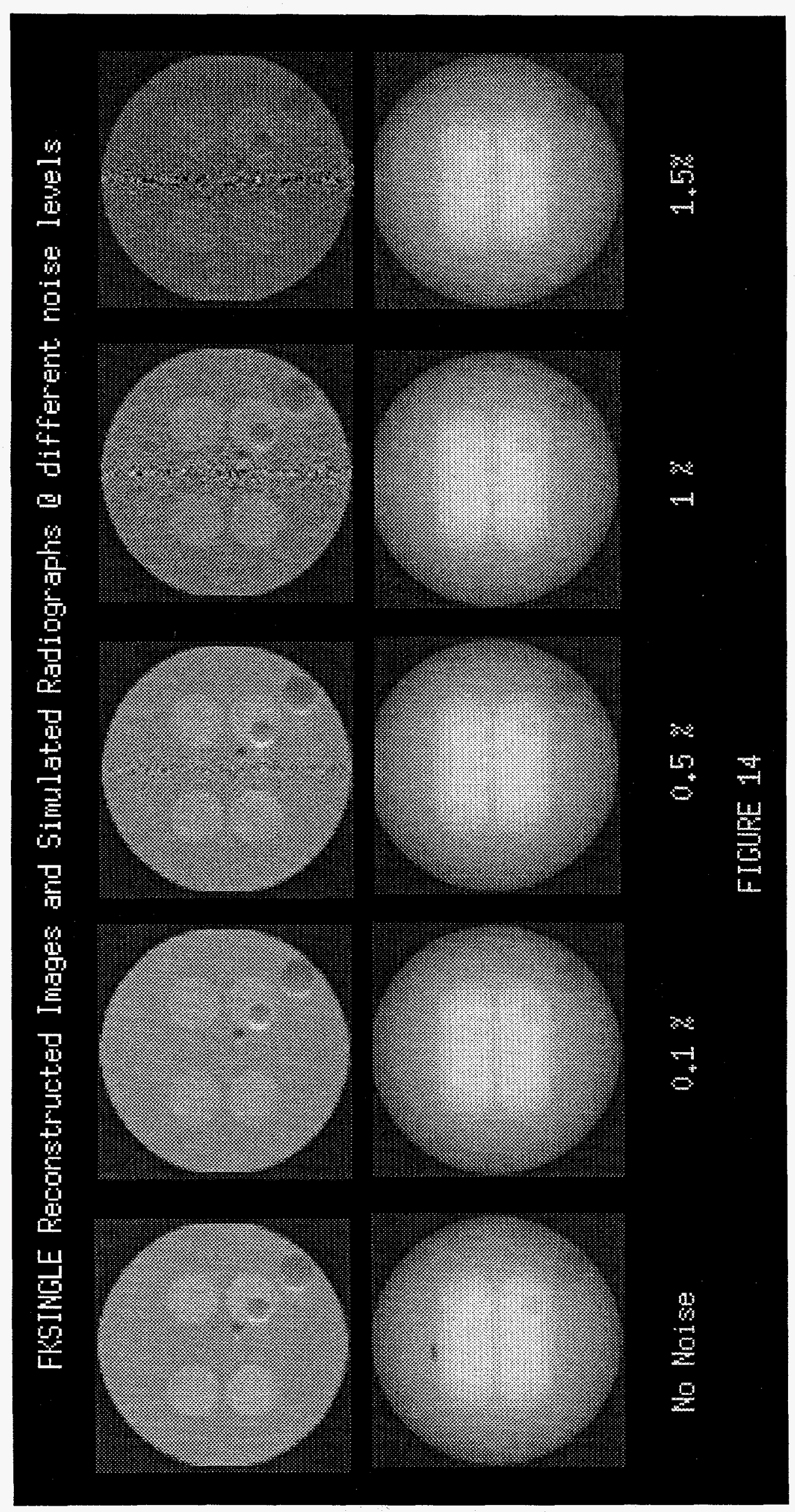




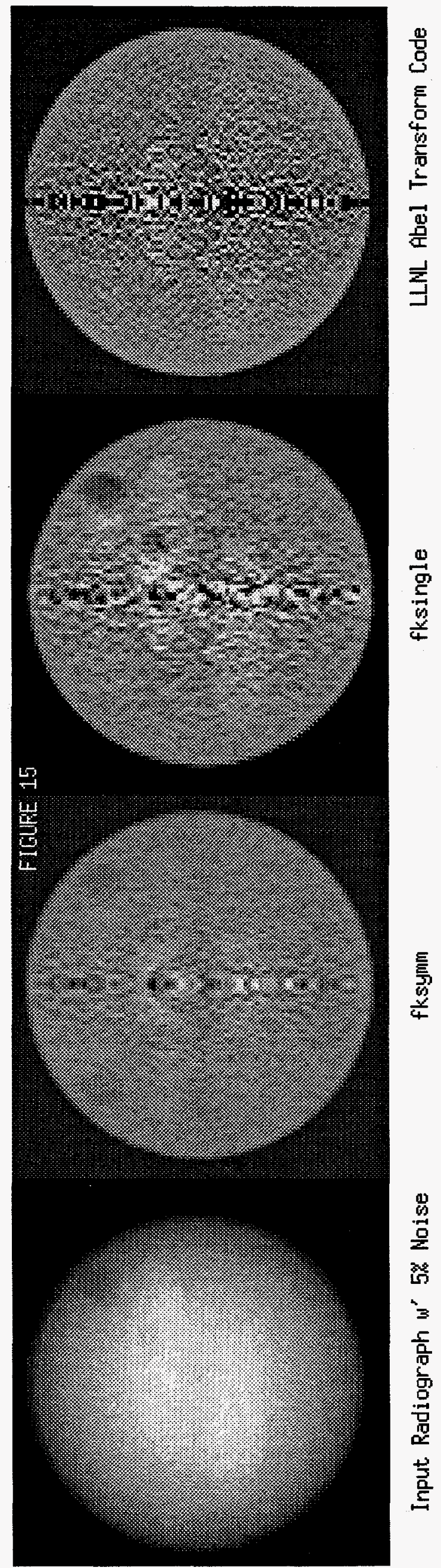




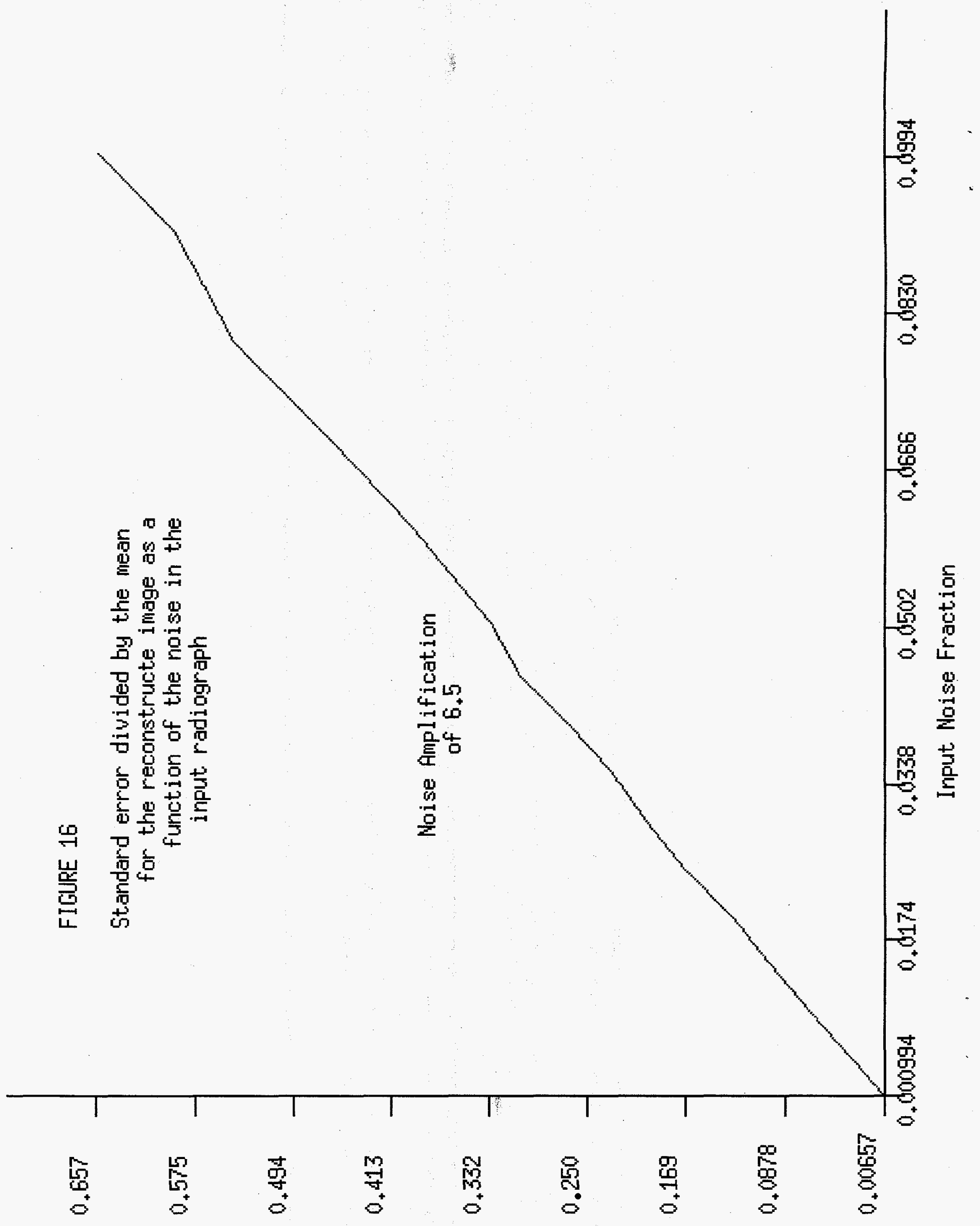



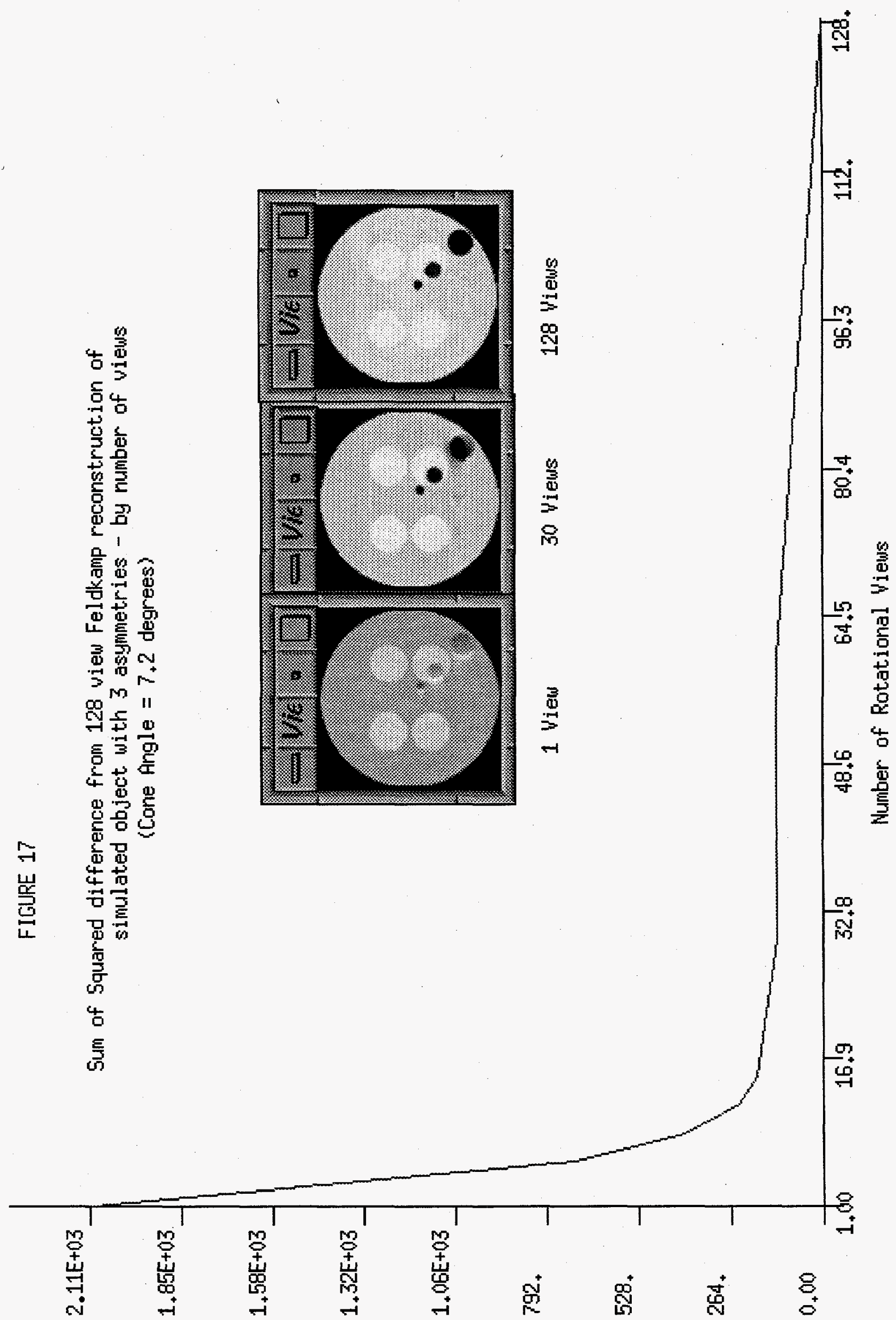


\section{(a) Core Punch Setup}
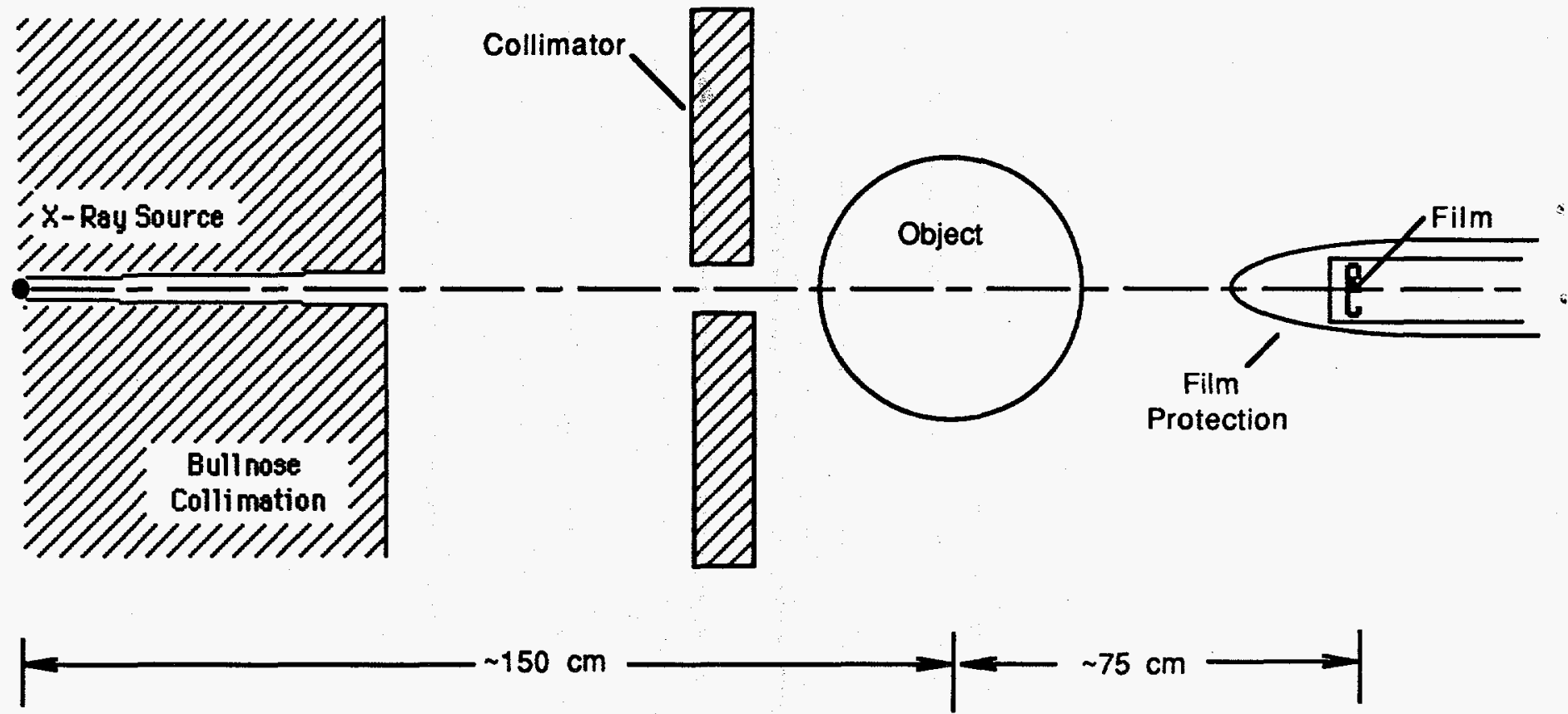

(b) Whole-Object Setup

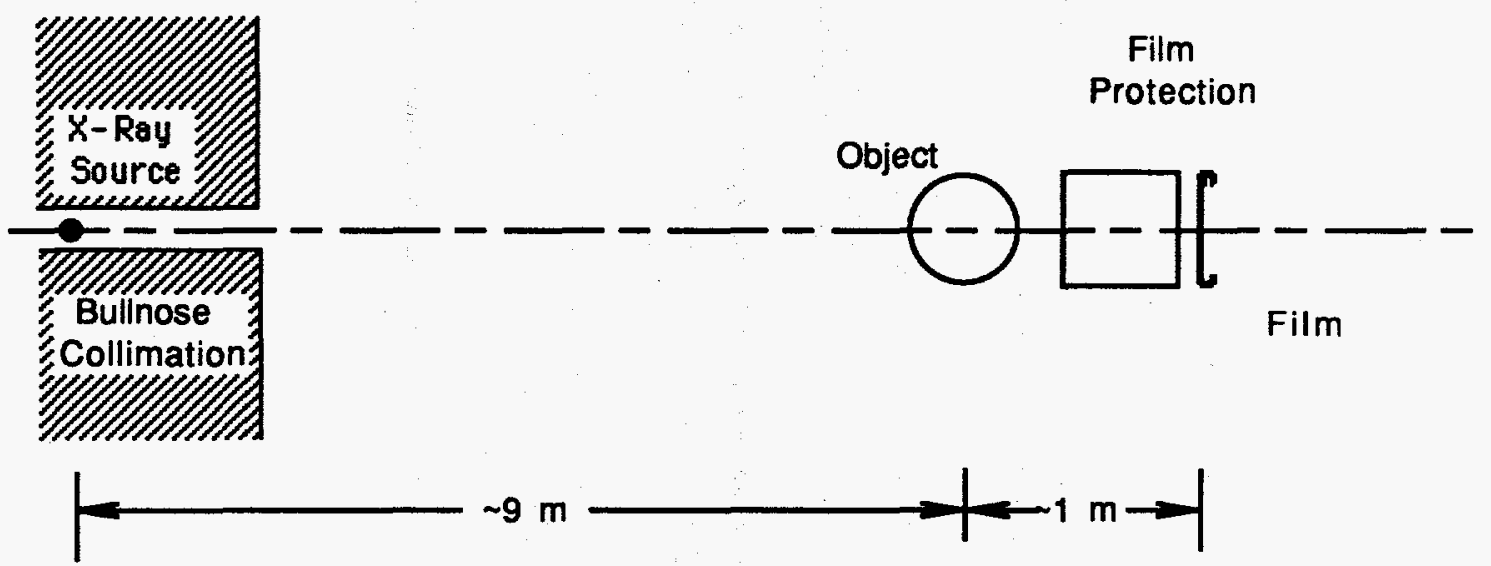

Figure 18 - Schematic representations (not to scale) of two typical types of FXR radiographic experiments: (a) a core punch, in which only the center of the object is imaged; (b) a wholeobject shot, in which the entire object is imaged 

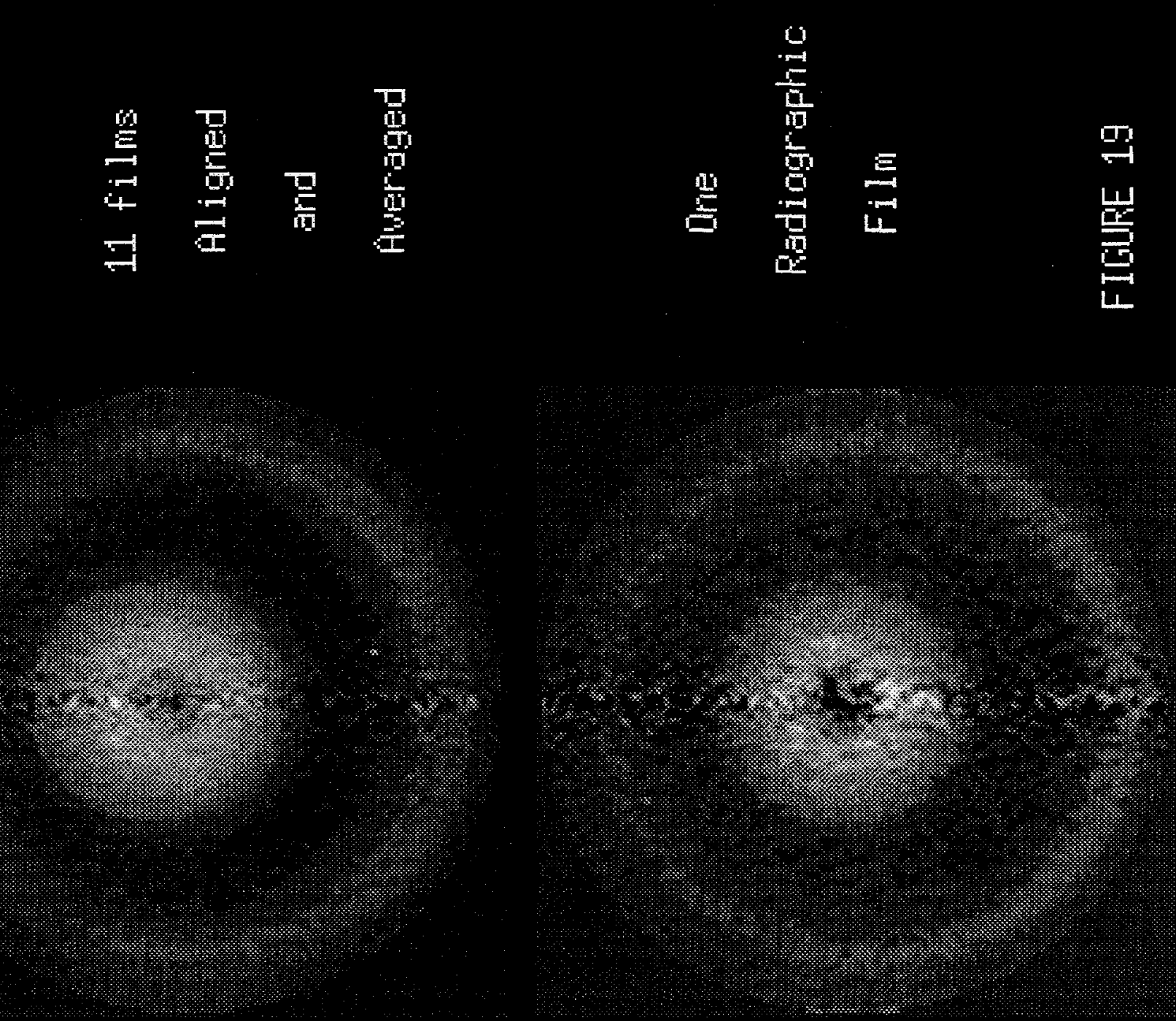

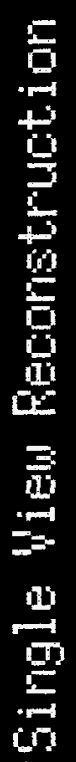

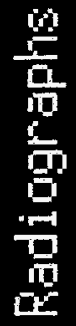


(a)

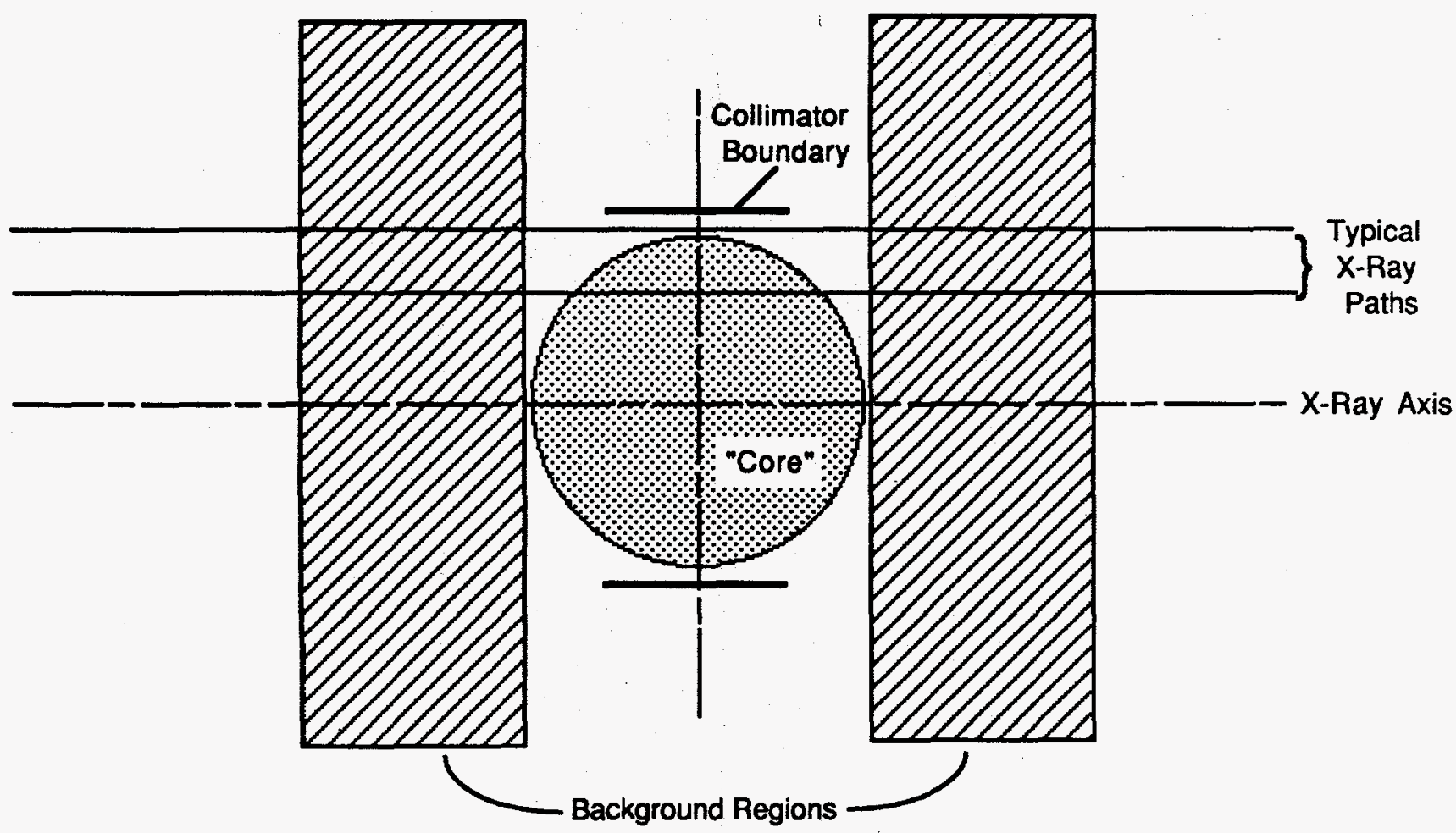

(b)

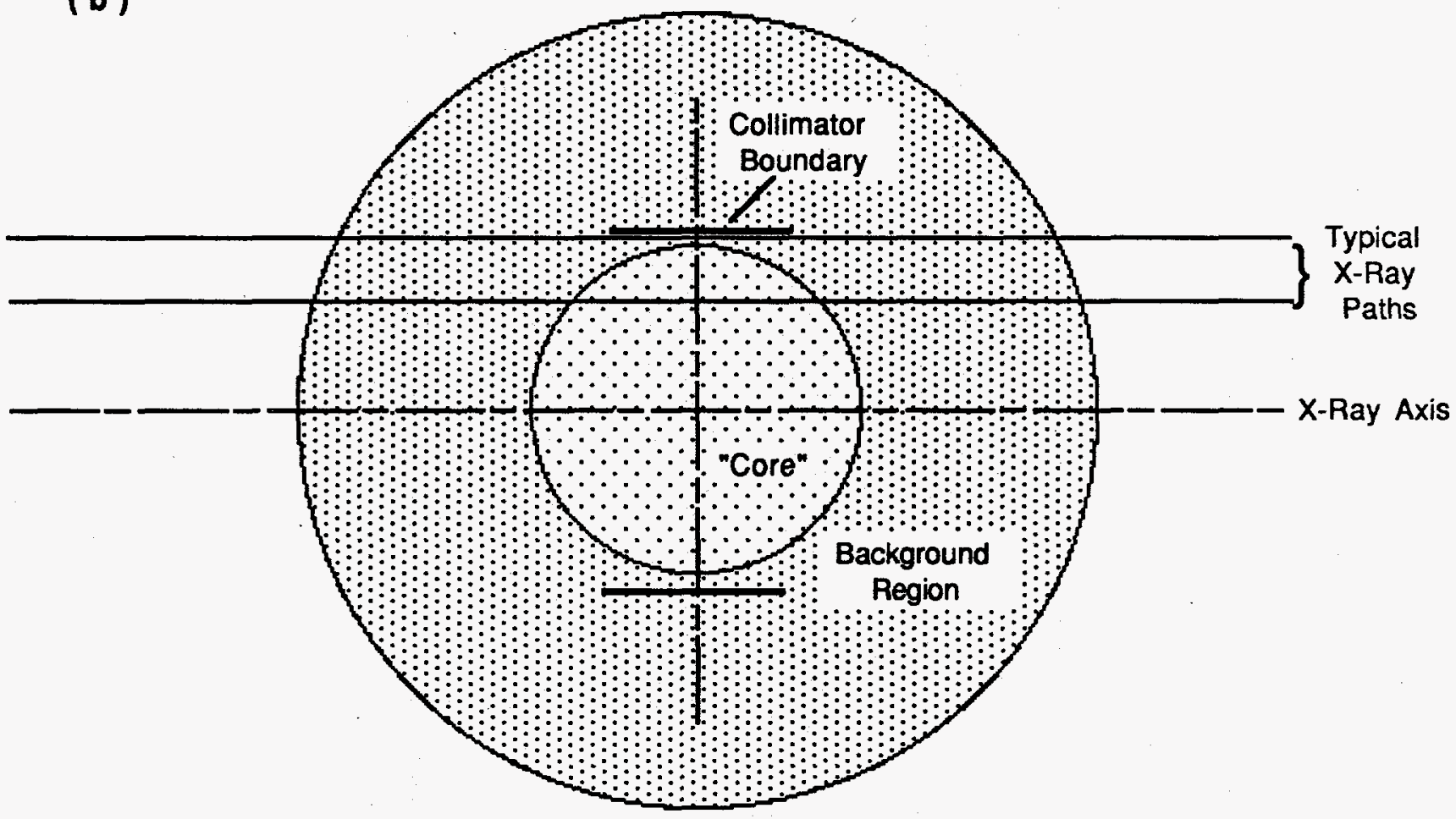

Figure 20 - Approximate methods for dealing with materials outside the collimated field of view ("background"): (a) assuming the background; (b) assuming the background is described accurately by a hydrocode calculation 